TRANSACTIONS OF THE

AMERICAN MATHEMATICAL SOCIETY

Volume 365, Number 7, July 2013, Pages 3851-3875

S 0002-9947(2012)05791-6

Article electronically published on December 17, 2012

\title{
REMARKS ON LAGRANGIAN INTERSECTIONS IN TORIC MANIFOLDS
}

\author{
MIGUEL ABREU AND LEONARDO MACARINI
}

\begin{abstract}
We consider two natural Lagrangian intersection problems in the context of symplectic toric manifolds: displaceability of torus orbits and of a torus orbit with the real part of the toric manifold. Our remarks address the fact that one can use simple cartesian product and symplectic reduction considerations to go from basic examples to much more sophisticated ones. We show in particular how rigidity results for the above Lagrangian intersection problems in weighted projective spaces can be combined with these considerations to prove analogous results for all monotone toric symplectic manifolds. We also discuss non-monotone and/or non-Fano examples, including some with a continuum of non-displaceable torus orbits.
\end{abstract}

\section{INTRODUCTION}

Let $\left(M^{2 n}, \omega\right)$ be a toric symplectic manifold, i.e. a symplectic manifold equipped with an effective Hamiltonian $\mathbb{T}^{n}$-action generated by a moment map

$$
\mu: M \rightarrow P:=\mu(M) \subset\left(\mathbb{R}^{n}\right)^{*},
$$

where the moment polytope $P$ is defined by

$$
\ell_{i}(x):=\left\langle x, \nu_{i}\right\rangle+a_{i} \geq 0, i=1, \ldots, d .
$$

Here the $a_{i}$ 's are real numbers, each vector $\nu_{i} \in \mathbb{Z}^{n}$ is the primitive integral interior normal to the facet $F_{i}$ of the polytope $P$ and $d$ is the number of facets of $P$.

Denote by $\tau: M \rightarrow M$ the canonical anti-symplectic involution, characterized by $\mu \circ \tau=\mu$, and let $R:=M^{\tau}$ denote its fixed point set. $R$ is a Lagrangian manifold, often called the real part of $M$.

Given $x \in \operatorname{int}(P)$, let $T_{x}:=\mu^{-1}(x)$ denote the corresponding $\mathbb{T}^{n}$-orbit, a Lagrangian torus in $M$. Since $\mu \circ \tau=\mu$, we also have that $\tau\left(T_{x}\right)=T_{x}$ and $\left(T_{x}\right)^{\tau}=T_{x} \cap R$. This last set, the real part of a regular $\mathbb{T}^{n}$-orbit, is discrete with $2^{n}$ points.

This can be seen quite explicitly in action-angle coordinates. Consider $\breve{M} \subset M$ defined by $\breve{M}=\mu^{-1}(\operatorname{int}(P))$. One checks that $\breve{M}$ is an open dense subset of $M$, consisting of all the points where the $\mathbb{T}^{n}$-action is free. It can be described as

$\breve{M} \cong \operatorname{int}(P) \times \mathbb{T}^{n}=\left\{\left(x_{1}, \ldots, x_{n}, e^{i \theta_{1}}, \ldots, e^{i \theta_{n}}\right): x \in \operatorname{int}(P) \subset \mathbb{R}^{n}, \theta \in \mathbb{R}^{n} / 2 \pi \mathbb{Z}^{n}\right\}$,

Received by the editors May 11, 2011 and, in revised form, December 5, 2011.

2010 Mathematics Subject Classification. Primary 53D12; Secondary 53D20.

The authors were partially supported by Fundação para a Ciência e a Tecnologia (FCT/Portugal), Fundação Coordenação de Aperfeiçoamento de Pessoal de Nível Superior (CAPES/Brazil) and Conselho Nacional de Desenvolvimento Científico e Tecnológico (CNPq/Brazil). 
where $(x, \theta)$ are symplectic action-angle coordinates for $\omega$, i.e.

$$
\left.\omega\right|_{\breve{M}}=d x \wedge d \theta=\sum_{j=1}^{n} d x_{j} \wedge d \theta_{j} .
$$

In these action-angle coordinates the moment map and anti-symplectic involution are given by

$$
\mu\left(x, e^{i \theta}\right)=x \quad \text { and } \quad \tau\left(x, e^{i \theta}\right)=\left(x, e^{-i \theta}\right) .
$$

Hence, we have that

$$
R \cap \breve{M}=(x, \pm 1) \equiv\left(x_{1}, \ldots, x_{n}, \pm 1, \ldots, \pm 1\right), x \in \operatorname{int}(P),
$$

and

$$
\sharp\left(R \pitchfork T_{x}\right)=2^{n}, \forall x \in \operatorname{int}(P) .
$$

Example 1.1. Consider $\left(\mathbb{C P}^{n}, \omega_{F S}\right)$ with moment polytope $P \subset\left(\mathbb{R}^{n}\right)^{*}$ given by

$$
P=\left\{\left(x_{1}, \ldots, x_{n}\right): x_{j}+1 \geq 0, j=1, \ldots, n, \text { and }-\left(\sum_{j=1}^{n} x_{j}\right)+1 \geq 0\right\} \text {. }
$$

Then

$$
R=\mathbb{R P}^{n} \quad \text { and } \quad T_{0}:=\mu^{-1}(0) \equiv \text { Clifford } n \text {-torus. }
$$

Example 1.2. Let $\left(M^{2 n}, \omega\right)$ be a monotone toric symplectic manifold, i.e. such that $[\omega]=\lambda\left(2 \pi c_{1}(\omega)\right) \in H^{2}(M)$ with $\lambda \in \mathbb{R}^{+}$. The corresponding moment polytope $P \subset\left(\mathbb{R}^{n}\right)^{*}$ is then a Fano Delzant polytope, meaning that it can be defined by (1) with

$$
a_{1}=\cdots=a_{d}=\lambda .
$$

In this case, the Lagrangian torus fiber over the origin $0 \in P$ will be called the centered or special torus fiber. It is the unique monotone torus fiber of the monotone toric symplectic manifold $\left(M^{2 n}, \omega\right)$.

Without any loss of generality, we will usually assume that $\lambda=1$ (as we already did in Example 1.1).

In this context of toric symplectic manifolds, it is natural to consider the following Lagrangian intersection rigidity question: given $x \in \operatorname{int}(P)$, does there exist $\psi \in$ $\operatorname{Ham}(M, \omega)$ such that

$$
\psi\left(T_{x}\right) \cap T_{x}=\emptyset \quad \text { or } \quad \psi\left(T_{x}\right) \cap R=\emptyset \quad \text { or } \quad \psi(R) \cap R=\emptyset ?
$$

Our remarks in this paper concern the first two of these Lagrangian intersection problems, and will show how simple cartesian product (Section 2) and symplectic reduction (Section 3) considerations can be used to go from basic examples to more sophisticated ones.

To illustrate our point, consider the following theorem on the most basic example that can be considered in this context.

Theorem 1.3. For $\left(\mathbb{C P}^{n}, \omega_{F S}\right)$, as in Example 1.1, we have that:

(i) [5, 8]

$$
\psi\left(T_{0}\right) \cap T_{0} \neq \emptyset \quad \text { and } \sharp\left(\psi\left(T_{0}\right) \pitchfork T_{0}\right) \geq 2^{n}, \forall \psi \in \operatorname{Ham}\left(\mathbb{C P}^{n}, \omega_{F S}\right) .
$$

(ii) [3, 4, 13, 20,

$$
\psi\left(T_{0}^{n}\right) \cap \mathbb{R} P^{n} \neq \emptyset, \forall \psi \in \operatorname{Ham}\left(\mathbb{C P}^{n}, \omega_{F S}\right) .
$$


(iii) [1]

$$
\sharp\left(\psi\left(T_{0}^{2 n-1}\right) \pitchfork \mathbb{R P}^{2 n-1}\right) \geq 2^{n}, \forall \psi \in \operatorname{Ham}\left(\mathbb{C P}^{2 n-1}, \omega_{F S}\right) .
$$

The following results follow by straightforward applications of our remarks.

Theorem 1.4 (Cf. Remark 2.10 and $\S$ 4.2).

$$
\sharp\left(\psi\left(T_{0}^{2 n}\right) \pitchfork \mathbb{R P}^{2 n}\right) \geq 2^{n}, \forall \psi \in \operatorname{Ham}\left(\mathbb{C P}^{2 n}, \omega_{F S}\right) .
$$

Theorem 1.5. Let $\left(M^{2 n}, \omega\right)$ be a monotone toric symplectic manifold, as in Example 1.2 , $R$ its real part and $T$ its special centered torus fiber. Let $\nu_{1}, \ldots, \nu_{d} \in \mathbb{Z}^{n}$ denote the primitive integral interior normals to the facets of the corresponding Fano Delzant polytope $P \subset\left(\mathbb{R}^{n}\right)^{*}$.

(i) (Cf. Proposition 4.4 in $\S 4.3$.) If $\sum_{i=1}^{d} \nu_{i}=0$, then

$$
\psi(T) \cap R \neq \emptyset, \psi(T) \cap T \neq \emptyset \quad \text { and } \sharp(\psi(T) \pitchfork T) \geq 2^{n}, \forall \psi \in \operatorname{Ham}(M, \omega) \text {. }
$$

(ii) (Cf. Proposition 4.6 in $\S$ 4.5.) If $P \subset\left(\mathbb{R}^{n}\right)^{*}$ is symmetric (i.e. whenever $\nu \in \mathbb{Z}^{n}$ is the normal to a facet of $P$, then $-\nu \in \mathbb{Z}^{n}$ is also the normal to a facet of $P$ ), then

$$
\sharp(\psi(T) \pitchfork R) \geq 2^{n}, \forall \psi \in \operatorname{Ham}(M, \omega) .
$$

Remark 1.6. A particularly interesting example that fits both (i) and (ii) of this theorem is $M=\mathbb{C P}^{2} \sharp 3 \overline{\mathbb{C P}}^{2}$, equipped with a monotone symplectic form (cf. $\S 4.5$ ).

Now consider the following generalization of part (i) of Theorem 1.3, contained in the work of Woodward [22] and of Cho and Poddar [10].

Theorem 1.7. Let $\mathbb{C P}(1, \mathbf{m}):=\mathbb{C P}\left(1, m_{1}, \ldots, m_{n}\right), m_{1}, \ldots, m_{n} \in \mathbb{N}$, denote the weighted projective space determined by the moment polytope $P_{\mathbf{m}} \subset\left(\mathbb{R}^{n}\right)^{*}$ given by

$$
P=\left\{\left(x_{1}, \ldots, x_{n}\right): x_{j}+1 \geq 0, j=1, \ldots, n, \text { and }-\left(\sum_{j=1}^{n} m_{j} x_{j}\right)+1 \geq 0\right\}
$$

(i.e. the symplectic quotient of $\mathbb{C}^{n+1}$ by the $S^{1}$-action with weights $\left(1, m_{1}, \ldots, m_{n}\right)$ ). Let $T_{0}:=\mu^{-1}(0)$ denote its special centered torus fiber, where $\mu: \mathbb{C P}(1, \mathbf{m}) \rightarrow P_{\mathbf{m}}$ is the moment map. Then

$$
\psi\left(T_{0}\right) \cap T_{0} \neq \emptyset \quad \text { and } \sharp\left(\psi\left(T_{0}\right) \pitchfork T_{0}\right) \geq 2^{n}, \forall \psi \in \operatorname{Ham}\left(\mathbb{C P}(1, \mathbf{m}), \omega_{\mathbf{m}}\right) .
$$

The following result, removing the zero-sum assumption in part (i) of Theorem 1.5 and originally due to Entov-Polterovich [13, Cho [9] and Fukaya-Oh-OhtaOno [15, follows from Theorem 1.7 by a straightforward application of our symplectic reduction remark (cf. Proposition 4.9] in $\S$ 4.7).

Theorem 1.8. Let $(M, \omega)$ be a compact monotone toric symplectic manifold, as in Example 1.2, and $T$ its special centered torus fiber. Then

$$
\psi(T) \cap T \neq \emptyset \quad \text { and } \quad \sharp(\psi(T) \pitchfork T) \geq 2^{n}, \forall \psi \in \operatorname{Ham}(M, \omega) .
$$

Remark 1.9. An appropriate generalization of part (ii) of Theorem 1.3 to weighted projective spaces would imply, by the same straightforward application of our symplectic reduction remark, that on any compact monotone toric symplectic manifold $(M, \omega)$ we also have that

$$
\psi(T) \cap R \neq \emptyset, \forall \psi \in \operatorname{Ham}(M, \omega) .
$$


This has been proved by Alston-Amorim [2] using the methods developed by Fukaya, Oh, Ohta and Ono in [14, 15, 16].

With the help of another basic example, i.e. the total space of the line bundle $\mathcal{O}(-1) \rightarrow \mathbb{C P}^{1}$ (cf. Section 5), our remarks can also be used to prove interesting non-displaceability results on certain non-monotone and/or non-Fano examples such as:

- a continuum of non-displaceable torus fibers on $M=\mathbb{C P}^{2} \sharp 2 \overline{\mathbb{C P}}^{2}$ with a certain non-monotone symplectic form (cf. Example 10.3 in [16] and $\S[5.4$ );

- a particular non-displaceable torus fiber on the family of non-Fano examples given by Hirzebruch surfaces $H_{k}:=\mathbb{P}(\mathcal{O}(-k) \oplus \mathbb{C}) \rightarrow \mathbb{C P}^{1}$, with $2 \leq k \in \mathbb{N}$ (cf. Example 10.1 in [16] and $\S 6.1$;

- a continuum of non-displaceable torus fibers on a certain non-Fano toric symplectic 4-manifold considered by McDuff (cf. $\S 2.1$ in [18] and $\S 6.2$ ).

For all non-displaceable torus fibers in these examples, we can also use our remarks to obtain an appropriate optimal lower bound for the number of transversal intersection points, which for these Lagrangian 2-tori is 4 .

Remark 1.10. Regarding this last example, McDuff shows in [18 that it gives rise, under a repeated wedge construction, to a monotone symplectic toric 12-manifold with a continuous interval of Lagrangian torus fibers that cannot be displaced by her method of probes [17. Hence, as she points out, these fibers "may perhaps be non-displaceable by Hamiltonian isotopies, even though, according to 14, their Floer homology vanishes". Although we can use the remarks in this paper to prove non-displaceability of the relevant torus fibers in the non-Fano toric symplectic 4-manifold (cf. $\S 6.2$ ), so far we have not been able to do the same for the corresponding monotone toric symplectic 12-manifold.

\section{First Remark: Cartesian product}

This first remark, on cartesian products, is motivated by Alston's result in part (iii) of Theorem 1.3 and can be used to remove its dimension restriction, i.e. prove Theorem 1.4. We will also use it in combination with our second remark, symplectic reduction.

2.1. Combinatorial Floer invariant. Let $P \subset\left(\mathbb{R}^{n}\right)^{*}$ be a moment polytope defined by

$$
\ell_{i}(x):=\left\langle x, \nu_{i}\right\rangle+a_{i} \geq 0, i=1, \ldots, d .
$$

Each vector $\nu_{i}=\left(\left(\nu_{i}\right)_{1}, \ldots,\left(\nu_{i}\right)_{n}\right) \in \mathbb{Z}^{n}$ is the primitive integral normal to the facet $F_{i}$ of the polytope $P$, and $d$ is the number of facets of $P$. We will say that $P$ is even if $d$ is even.

Let $C F^{n}$ be the vector space of dimension $2^{n}$ generated over $\mathbb{Z}_{2}$ by the following $2^{n}$ symbols:

$$
\left(\varepsilon_{1}, \ldots, \varepsilon_{n}\right) \text { with } \varepsilon_{k}= \pm 1, k=1, \ldots, n .
$$

Consider the linear map

$$
\partial_{P}: C F^{n} \rightarrow C F^{n}
$$

defined on basis elements by

$$
\partial_{P}(\varepsilon)=\sum_{i=1}^{d}(-1)^{\nu_{i}} \varepsilon
$$


where $\varepsilon=\left(\varepsilon_{1}, \ldots, \varepsilon_{n}\right)$ and

$$
(-1)^{\nu_{i}} \varepsilon=\left((-1)^{\left(\nu_{i}\right)_{1}} \varepsilon_{1}, \ldots,(-1)^{\left(\nu_{i}\right)_{n}} \varepsilon_{n}\right) .
$$

Proposition 2.2. $\partial_{P}^{2}=0$ if $d$ is even and $\partial_{P}^{2}=$ id if $d$ is odd.

Proof.

$$
\begin{aligned}
\partial_{P}\left(\partial_{P} \varepsilon\right) & =\partial_{P}\left(\sum_{i=1}^{d}(-1)^{\nu_{i}} \varepsilon\right)=\sum_{i=1}^{d} \partial_{P}\left((-1)^{\nu_{i}} \varepsilon\right) \\
& =\sum_{i, j=1}^{d}(-1)^{\nu_{j}}\left((-1)^{\nu_{i}} \varepsilon\right)=\sum_{i, j=1}^{d}(-1)^{\nu_{i}+\nu_{j}} \varepsilon \\
& =2\left(\sum_{i<j}(-1)^{\nu_{i}+\nu_{j}} \varepsilon\right)+\sum_{i=1}^{d}(-1)^{2 \nu_{i}} \varepsilon \\
& =0+\sum_{i=1}^{d} \varepsilon=d \varepsilon .
\end{aligned}
$$

Definition 2.3. The Floer invariant $H F(P)$ of an even integral polytope $P$ is defined by

$$
H F(P):=\operatorname{dim} \operatorname{ker}\left(\partial_{P}\right)-\operatorname{dim} \operatorname{im}\left(\partial_{P}\right) .
$$

Proposition 2.4. If $P=P^{\prime} \times P^{\prime \prime}$, with $P^{\prime}$ and $P^{\prime \prime}$ even integral polytopes, then

$$
H F(P)=H F\left(P^{\prime}\right) \cdot H F\left(P^{\prime \prime}\right) .
$$

Proof. Suppose $P^{\prime} \subset \mathbb{R}^{n^{\prime}}$ and $P^{\prime \prime} \subset \mathbb{R}^{n^{\prime \prime}}$ have normal vectors

$$
\nu_{1}^{\prime}, \ldots, \nu_{d^{\prime}}^{\prime} \in \mathbb{Z}^{n^{\prime}} \quad \text { and } \quad \nu_{1}^{\prime \prime}, \ldots, \nu_{d^{\prime \prime}}^{\prime \prime} \in \mathbb{Z}^{n^{\prime \prime}} .
$$

Then $P=P^{\prime} \times P^{\prime \prime} \subset \mathbb{R}^{n^{\prime}} \times \mathbb{R}^{n^{\prime \prime}}=\mathbb{R}^{n}, n=n^{\prime}+n^{\prime \prime}$, has normal vectors

$$
\left(\nu_{1}^{\prime}, 0\right), \ldots,\left(\nu_{d^{\prime}}^{\prime}, 0\right),\left(0, \nu_{1}^{\prime \prime}\right), \ldots,\left(0, \nu_{d^{\prime \prime}}^{\prime \prime}\right) \in \mathbb{Z}^{n^{\prime}} \times \mathbb{Z}^{n^{\prime \prime}}=\mathbb{Z}^{n} .
$$

Hence, the linear map $\partial_{P}$ on $C F^{n}=C F^{n^{\prime}} \otimes C F^{n^{\prime \prime}}$ is given by

$$
\partial_{P}\left(\varepsilon^{\prime} \otimes \varepsilon^{\prime \prime}\right)=\left(\partial_{P^{\prime}} \varepsilon^{\prime}\right) \otimes \varepsilon^{\prime \prime}+\varepsilon^{\prime} \otimes\left(\partial_{P^{\prime \prime}} \varepsilon^{\prime \prime}\right) .
$$

The result of the proposition follows by a standard application of the Künneth formula in this context.

Using this proposition and the fact that $P \times P$ always has an even number of facets, we can define the Floer invariant of any integral polytope.

Definition 2.5. The Floer invariant $H F(P)$ of an integral polytope $P$ is defined by

$$
H F(P):=\sqrt{\operatorname{dim} \operatorname{ker}\left(\partial_{P \times P}\right)-\operatorname{dim} \operatorname{im}\left(\partial_{P \times P}\right)} .
$$


2.6. Relation with Lagrangian Floer homology. Now suppose that $P$ is a Fano Delzant polytope and let $M_{P}$ denote its associated smooth Fano toric variety. This means that when defining $P \subset\left(\mathbb{R}^{n}\right)^{*}$ by

$$
\ell_{i}(x):=\left\langle x, \nu_{i}\right\rangle+a_{i} \geq 0, i=1, \ldots, d,
$$

we can assume that $a_{1}=\cdots=a_{d}=1$ and $\left(M_{P}, \omega_{P}\right)$ is monotone: $\left[\omega_{P}\right]=$ $2 \pi c_{1}\left(M_{P}\right)$.

Denote by $R_{P}$ the Lagrangian real part of $\left(M_{P}, \omega_{P}\right)$ and by $T_{P}$ the Lagrangian torus over $0 \in \operatorname{int}(P)$. Assuming the Lagrangian Floer homology $\operatorname{HF}\left(R_{P}, T_{P} ; \mathbb{Z}_{2}\right)$ is well defined, we have the following theorem.

\section{Theorem 2.7.}

$$
\operatorname{dim} H F\left(R_{P}, T_{P} ; \mathbb{Z}_{2}\right)=H F(P) .
$$

\section{Corollary 2.8.}

$$
\sharp\left(\psi\left(R_{P}\right) \pitchfork T_{P}\right) \geq H F(P), \forall \psi \in \operatorname{Ham}\left(M_{P}, \omega_{P}\right) .
$$

Proof of the corollary. If $P$ is a Fano Delzant polytope, then $P \times P$ is an even Fano Delzant polytope to which Theorem 2.7 applies. Suppose that

$$
\sharp\left(\psi\left(R_{P}\right) \cap T_{P}\right)<H F(P) .
$$

Then $(\psi \times \psi) \in \operatorname{Ham}\left(M_{P} \times M_{P}, \omega_{P} \times \omega_{P}\right)$ would be such that

$\sharp\left[(\psi \times \psi)\left(R_{P} \times R_{P}\right) \cap\left(T_{P} \times T_{P}\right)\right]=\left(\sharp\left(\psi\left(R_{P}\right) \cap T_{P}\right)\right)^{2}<(H F(P))^{2}=H F(P \times P)$,

which would imply that

$$
\operatorname{dim} H F\left(R_{P \times P}, T_{P \times P} ; \mathbb{Z}_{2}\right)<H F(P \times P) .
$$

This contradicts Theorem 2.7. As before, we are assuming $H F\left(R_{P \times P}, T_{P \times P} ; \mathbb{Z}_{2}\right)$ is well defined.

Remark 2.9. The meaning of $H F\left(R_{P}, T_{P} ; \mathbb{Z}_{2}\right)$ being well defined depends on the technical tools one is willing to use. If one uses only a basic version of Lagrangian Floer homology, then the condition that the minimal Maslov number of $R_{P}$ is larger than two has to be enforced. Under this assumption, it can be proved as in [1, 8, 11, 19] that, for the standard complex structure $J$ on $M_{P}$, the boundary of the moduli space of holomorphic strips with dimension one is given by broken strips and holomorphic disks with Maslov index two and boundary in $T_{P}$. Moreover, the linearized Cauchy-Riemann operator $D_{u} \bar{\partial}_{J}$ is surjective for all $J$-holomorphic strips $u$ of Maslov index one or two. It follows from this that $H F\left(R_{P}, T_{P} ; \mathbb{Z}_{2}\right)$ is well defined whenever $d$ is even. As a matter of fact, if $d$ is even, then the holomorphic disks with index two and boundary in $T_{P}$ counted by $\partial_{P}^{2}$ cancel out when we work with $\mathbb{Z}_{2}$ coefficients; this is the key point in the proof of Proposition 2.2. In particular, $H F\left(R_{P \times P}, T_{P \times P} ; \mathbb{Z}_{2}\right)$ is well defined whatever the parity of $d$ may be. Indeed, the minimal Maslov number of $R_{P \times P}$ equals the minimal Maslov number of $R_{P}$.

With this hypothesis on $R_{P}$ the set of examples that are covered by Corollary 2.8 is a bit restrictive. If one uses a sophisticated version of Lagrangian Floer homology, such as the one developed by Fukaya, Oh, Ohta and Ono in [14, 15, 16, then Corollary 2.8 covers much more ground (see [2]). 
Remark 2.10. Even with only basic Lagrangian Floer homology, we see that $H F\left(R_{P \times P}, T_{P \times P} ; \mathbb{Z}_{2}\right)$ is indeed well defined when $P$ is a simplex, i.e. $M_{P}=\mathbb{C P}^{n}$, and Corollary 2.8 can be combined with a combinatorial computation of $H F(P \times P)$ to prove Theorem 1.4 hence removing the dimension restriction of Alston's result. We will not present that combinatorial computation here since (i) it is presented in [2] and (ii), as we will see, our remark on symplectic reduction can also be used to easily remove this restriction.

The proof of Theorem 2.7 is a simple combination of the following two ingredients:

(i) the characterization by Cho 8 and Cho-Oh [11] of the holomorphic discs on Fano toric manifolds, that are relevant for the differential on the Lagrangiam Floer complex, as Blaschke products;

(ii) the existence of global homogeneous coordinates on any smooth toric variety $M_{P}$, not just on $\mathbb{C} P^{n}$ (see $\S 4.4$ of Cox [12]).

We will now discuss some details of (ii) and how they contribute to the proof of Theorem 2.7

From the well-known quotient representation

$$
M_{P}=\left(\mathbb{C}^{d} \backslash Z_{P}\right) / G_{P}
$$

we get a map

$$
\begin{aligned}
\mathbb{C}^{d} \backslash Z_{P} & \longrightarrow M_{P}, \\
\left(z_{1}, \ldots, z_{d}\right) & \longmapsto\left[z_{1}, \ldots, z_{d}\right]
\end{aligned}
$$

defining homogeneous coordinates on $M_{P}$, where here homogeneous is with respect to the action of the torus $G_{P}$. Note that the canonical anti-holomorphic involution $\tau_{P}: M_{P} \rightarrow M_{P}$ is given in these homogeneous coordinates as

$$
\tau_{P}\left(\left[z_{1}, \ldots, z_{d}\right]\right)=\left[\bar{z}_{1}, \ldots, \bar{z}_{d}\right] .
$$

Let $m_{1}, \ldots, m_{\ell}$ be the integral points in $P$, i.e.

$$
\left\{m_{1}, \ldots, m_{\ell}\right\}=P \cap \mathbb{Z}^{n},
$$

and consider the map

$$
\phi: \mathbb{C}^{d} \backslash Z_{P} \rightarrow \mathbb{C} P^{\ell-1}
$$

given by

$$
\phi\left(z_{1}, \ldots, z_{d}\right)=\left[z^{\left[m_{1}\right]}, \ldots, z^{\left[m_{\ell}\right]}\right]
$$

where

$$
z^{[m]}=\prod_{i=1}^{d} z_{i}^{\ell_{i}(m)} .
$$

It turns out that $\phi$ induces a well-defined embedding

$$
\begin{aligned}
M_{P} & \longrightarrow \mathbb{C} P^{\ell-1}, \\
{\left[z_{1}, \ldots, z_{d}\right] } & \longmapsto\left[z^{\left[m_{1}\right]}, \ldots, z^{\left[m_{\ell}\right]}\right] .
\end{aligned}
$$

If one restricts this map to $\left(\mathbb{C}^{*}\right)^{n} \subset M_{P}$, one gets an embedding

$$
\varphi:\left(\mathbb{C}^{*}\right)^{n} \rightarrow \mathbb{C} P^{\ell-1}
$$

given in the standard coordinates of $\left(\mathbb{C}^{*}\right)^{n}$ as

$$
\varphi\left(t_{1}, \ldots, t_{n}\right)=\left[t^{m_{1}}, \ldots, t^{m_{\ell}}\right],
$$


where

$$
t^{m_{k}}=\prod_{i=1}^{n} t_{i}^{\left(m_{k}\right)_{i}}
$$

Since

$$
R_{P} \cap\left(\mathbb{C}^{*}\right)^{n}=\left(\mathbb{R}^{*}\right)^{n} \quad \text { and } \quad T_{P}=\left(S^{1}\right)^{n} \subset\left(\mathbb{C}^{*}\right)^{n}
$$

we get that

$$
R_{P} \cap T_{P}=\left\{\left(\varepsilon_{1}, \ldots, \varepsilon_{n}\right): \varepsilon_{i}= \pm 1\right\} .
$$

Any such intersection point can be written in homogeneous coordinates as

$$
\left[\varepsilon_{1}, \ldots, \varepsilon_{d}\right], \varepsilon_{i}= \pm 1 \text {. }
$$

As Alston [1] does in the case of $\mathbb{C} P^{n}$, using the work of Cho [8] and Cho-Oh 11, the characterization of relevant holomorphic discs as Blaschke products shows that the Lagrangian Floer differential is given in homogeneous coordinates by

$$
\partial_{P}\left(\left[\varepsilon_{1}, \ldots, \varepsilon_{d}\right]\right)=\sum_{i=1}^{d}\left[\varepsilon_{1}, \ldots,-\varepsilon_{i}, \ldots, \varepsilon_{d}\right] .
$$

To compute $\partial_{P}\left(\varepsilon_{1}, \ldots, \varepsilon_{n}\right)$ directly, we can now use the maps $\phi$ and $\varphi$, defined by (3) and (4). Note that if

$$
\phi\left(\left[\varepsilon_{1}, \ldots, \varepsilon_{i}, \ldots, \varepsilon_{d}\right]\right)=\left[\varepsilon^{\left[m_{1}\right]}, \ldots, \varepsilon^{\left[m_{\ell}\right]}\right],
$$

then

$$
\begin{aligned}
\phi\left(\left[\varepsilon_{1}, \ldots,-\varepsilon_{i}, \ldots, \varepsilon_{d}\right]\right) & =\left[(-1)^{\ell_{i}\left(m_{1}\right)} \varepsilon^{\left[m_{1}\right]}, \ldots,(-1)^{\ell_{i}\left(m_{\ell}\right)} \varepsilon^{\left[m_{\ell}\right]}\right] \\
& =\left[(-1)^{\left\langle m_{1}, \nu_{i}\right\rangle+a_{i}} \varepsilon^{\left[m_{1}\right]}, \ldots,(-1)^{\left\langle m_{\ell}, \nu_{i}\right\rangle+a_{i}} \varepsilon^{\left[m_{\ell}\right]}\right] \\
& =\left[(-1)^{\left\langle m_{1}, \nu_{i}\right\rangle} \varepsilon^{\left[m_{1}\right]}, \ldots,(-1)^{\left\langle m_{\ell}, \nu_{i}\right\rangle} \varepsilon^{\left[m_{\ell}\right]}\right] .
\end{aligned}
$$

On the other hand, if

$$
\varphi\left(t_{1}, \ldots, t_{n}\right)=\left[t^{m_{1}}, \ldots, t^{m_{\ell}}\right]=\left[\varepsilon^{\left[m_{1}\right]}, \ldots, \varepsilon^{\left[m_{\ell}\right]}\right],
$$

then

$$
\begin{aligned}
\varphi\left((-1)^{\left(\nu_{i}\right)_{1}} t_{1}, \ldots,(-1)^{\left(\nu_{i}\right)_{n}} t_{n}\right) & =\left[(-1)^{\left\langle m_{1}, \nu_{i}\right\rangle} t^{m_{1}}, \ldots,(-1)^{\left\langle m_{\ell}, \nu_{i}\right\rangle} t^{m_{\ell}}\right] \\
& =\left[(-1)^{\left\langle m_{1}, \nu_{i}\right\rangle} \varepsilon^{\left[m_{1}\right]}, \ldots,(-1)^{\left\langle m_{\ell}, \nu_{i}\right\rangle} \varepsilon^{\left[m_{\ell}\right]}\right] \\
& =\phi\left(\left[\varepsilon_{1}, \ldots,-\varepsilon_{i}, \ldots, \varepsilon_{d}\right]\right) .
\end{aligned}
$$

Hence,

$$
\begin{aligned}
\partial_{P}\left(\left[\varepsilon_{1}, \ldots, \varepsilon_{d}\right]\right) & =\sum_{i=1}^{d}\left[\varepsilon_{1}, \ldots,-\varepsilon_{i}, \ldots, \varepsilon_{d}\right] \\
\Leftrightarrow \quad \partial_{P}\left(\varepsilon_{1}, \ldots, \varepsilon_{n}\right) & =\sum_{i=1}^{d}\left((-1)^{\left(\nu_{i}\right)_{1}} \varepsilon_{1}, \ldots,(-1)^{\left(\nu_{i}\right)_{n}} \varepsilon_{n}\right) .
\end{aligned}
$$




\subsection{Examples.}

Example 2.12. Let $P=[-1,1] \subset \mathbb{R}$ with $M_{P}=\mathbb{C} P^{1}=S^{2}$. In this case $R_{P}$ is a meridian circle and $T_{P}$ is the equator. $P$ has normals $\nu_{1}=1$ and $\nu_{2}=-1$. $C F$ is a 2-dimensional vector space with basis $e_{1}=(1)$ and $e_{2}=(-1)$. The differential $\partial_{P}$ is given by

$$
\partial_{P}(\varepsilon)=\left((-1)^{1} \varepsilon\right)+\left((-1)^{-1} \varepsilon\right)=2(-\varepsilon)=0 .
$$

Hence,

$$
H F(P)=2 .
$$

Proposition 2.13. If $P$ is an even polytope such that, whenever $\nu$ is a normal, $-\nu$ is also a normal, i.e. such that $P$ is symmetric, then $H F(P)=2^{n}$.

Proof. As in the example above, we always have $\partial_{P}=0$ under these conditions.

Remark 2.14. Under the assumption that $H F\left(R_{P}, T_{P} ; \mathbb{Z}_{2}\right)$ is well defined, it follows from this proposition that if $P$ is symmetric, then

$$
\sharp\left(\psi\left(T_{P}\right) \cap R_{P}\right) \geq 2^{n} \quad \text { for any } \psi \in \operatorname{Ham}\left(M_{P}, \omega_{P}\right) .
$$

In fact, as we will see in Proposition 4.6, one can easily use our remark on symplectic reduction to remove the assumption and give another proof of this result.

Example 2.15. When $n=2$ there are exactly two even Fano polytopes with the property of Proposition 2.13, the Fano square, corresponding to $\mathbb{C} P^{1} \times \mathbb{C} P^{1}$, and the Fano hexagon, corresponding to $\mathbb{C} P^{2}$ blown up at 3 points.

Example 2.16. Let $P \subset \mathbb{R}^{2}$ be the Fano simplex corresponding to $M_{P}=\mathbb{C} P^{2}$. Since $P$ has 3 facets, an odd number, we will consider the even Fano Delzant polytope $P \times P \subset \mathbb{R}^{4}$, whose 6 facets have normals

$$
\begin{gathered}
\nu_{1}=(1,0,0,0), \nu_{2}=(0,1,0,0), \nu_{3}=(-1,-1,0,0), \nu_{4}=(0,0,1,0), \\
\nu_{5}=(0,0,0,1) \text { and } \nu_{6}=(0,0,-1,-1) .
\end{gathered}
$$

This means that $\partial_{P \times P}: C F^{4} \rightarrow C F^{4}$ is given by

$$
\begin{aligned}
\partial_{P \times P}\left(\varepsilon_{1}, \varepsilon_{2}, \varepsilon_{3}, \varepsilon_{4}\right) & =\left(-\varepsilon_{1}, \varepsilon_{2}, \varepsilon_{3}, \varepsilon_{4}\right)+\left(\varepsilon_{1},-\varepsilon_{2}, \varepsilon_{3}, \varepsilon_{4}\right)+\left(-\varepsilon_{1},-\varepsilon_{2}, \varepsilon_{3}, \varepsilon_{4}\right) \\
& +\left(\varepsilon_{1}, \varepsilon_{2},-\varepsilon_{3}, \varepsilon_{4}\right)+\left(\varepsilon_{1}, \varepsilon_{2}, \varepsilon_{3},-\varepsilon_{4}\right)+\left(\varepsilon_{1}, \varepsilon_{2},-\varepsilon_{3},-\varepsilon_{4}\right) .
\end{aligned}
$$

With this explicit formula it is not hard to check that

$$
H F(P \times P)=\operatorname{dim} \operatorname{ker}\left(\partial_{P \times P}\right)-\operatorname{dim} \operatorname{im}\left(\partial_{P \times P}\right)=10-6=4 .
$$

Hence,

$$
H F(P)=\sqrt{4}=2,
$$

and applying Corollary 2.8 we conclude that

$$
\sharp\left(\psi\left(\mathbb{R} P^{2}\right) \cap \mathbb{T}^{2}\right) \geq 2,
$$

for any $\psi \in \operatorname{Ham}\left(\mathbb{C} P^{2}\right)$. This estimate is known to be optimal (see for example the end of section 5 in $[2]$ ). 


\section{SECOND REMARK: Symplectic REDUCTION}

Here we will state some elementary general facts in the particular context of symplectic toric manifolds.

Let $(\tilde{M}, \tilde{\omega})$ be a symplectic toric manifold of dimension $2 N$ with $\tilde{\mathbb{T}}$-action generated by a moment map

$$
\tilde{\mu}: \tilde{M} \rightarrow \tilde{P} \subset\left(\mathbb{R}^{N}\right)^{*} .
$$

As before, given $x \in \operatorname{int}(\tilde{P})$, let $\tilde{T}_{x}:=\tilde{\mu}^{-1}(x)$ denote the corresponding $\tilde{\mathbb{T}}$-orbit, a Lagrangian torus in $\tilde{M}$, and let $\tilde{R}$ denote the real part of $\tilde{M}$, i.e. the Lagrangian submanifold given by the fixed point set of the canonical anti-symplectic involution $\tilde{\tau}: \tilde{M} \rightarrow \tilde{M}$, characterized by $\tilde{\mu} \circ \tilde{\tau}=\tilde{\mu}$. Recall that

$$
\tilde{\tau}(\tilde{\theta} \cdot \tilde{p})=-\tilde{\theta} \cdot(\tilde{\tau}(\tilde{p})), \forall \tilde{\theta} \in \tilde{\mathbb{T}}, \tilde{p} \in \tilde{M}, \quad \text { and } \quad \tilde{\tau}\left(\tilde{T}_{x}\right)=\tilde{T}_{x} .
$$

Moreover, $\left(\tilde{T}_{x}\right)^{\tilde{\tau}}=\tilde{T}_{x} \cap \tilde{R}$, and this set, the real part of a regular $\tilde{\mathbb{T}}$-orbit, is discrete with $2^{N}$ points.

Let $K \subset \tilde{\mathbb{T}}$ be a subtorus of dimension $N-n$ determined by an inclusion of Lie algebras

$$
\iota: \mathbb{R}^{N-n} \rightarrow \mathbb{R}^{N}
$$

The moment map for the induced action of $K$ on $\tilde{M}$ is

$$
\tilde{\mu}_{K}=\iota^{*} \circ \tilde{\mu}: \tilde{M} \rightarrow\left(\mathbb{R}^{N-n}\right)^{*} .
$$

Let $c \in \tilde{\mu}_{K}(\tilde{M}) \subset\left(\mathbb{R}^{N-n}\right)^{*}$ be a regular value and assume that $K$ acts freely on the level set

$$
Z:=\tilde{\mu}_{K}^{-1}(c) \subset \tilde{M} .
$$

Then, the reduced space $(M:=Z / K, \omega)$ is a symplectic toric manifold of dimension $2 n$ with $\mathbb{T}:=\tilde{\mathbb{T}} / K$-action generated by a moment map

$$
\mu: M \rightarrow P \subset\left(\mathbb{R}^{n}\right)^{*} \cong \operatorname{ker}\left(\iota^{*}\right)
$$

that fits in the commutative diagram

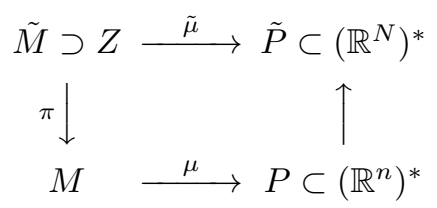

where $\pi$ is the quotient projection and the vertical arrow on the right is the inclusion $\left(\mathbb{R}^{n}\right)^{*} \cong \operatorname{ker}\left(\iota^{*}\right) \subset\left(\mathbb{R}^{N-n}\right)^{*}$.

Recall that the reduced symplectic form $\omega$ is characterized by $\pi^{*} \omega=\left.\tilde{\omega}\right|_{Z}$. Note that given $T_{x}:=\mu^{-1}(x)$, with $x \in \operatorname{int}(P) \subset \operatorname{int}(\tilde{P})$, we have that

$$
\pi^{-1}\left(T_{x}\right)=\tilde{T}_{x}
$$

Moreover, $\tilde{\tau}(Z)=Z, Z^{\tilde{\tau}}=Z \cap \tilde{R}$ and $\tilde{\tau}$ induces the canonical anti-symplectic involution $\tau: M \rightarrow M$ via

$$
\pi \circ \tilde{\tau}=\tau \circ \pi .
$$

Let $p \in R:=M^{\tau}$. Then a simple counting argument shows that

$$
\sharp\left(\pi^{-1}(p) \cap \tilde{R}\right)=2^{N-n} .
$$

Lemma 3.1. Let $\psi \in \operatorname{Ham}(M, \omega)$. Then there is $\tilde{\psi} \in \operatorname{Ham}(\tilde{M}, \tilde{\omega})$ such that $\tilde{\psi}(Z)=$ $Z$ and

$$
\pi(\tilde{\psi}(\tilde{p}))=\psi(\pi(\tilde{p})), \forall \tilde{p} \in Z
$$


Proof. Given a time dependent Hamiltonian $h_{t}: M \rightarrow \mathbb{R}$, let $\tilde{h}_{t}: \tilde{M} \rightarrow \mathbb{R}$ be any smooth extension to $\tilde{M}$ of $h_{t} \circ \pi: Z \rightarrow \mathbb{R}$. The Hamiltonian flow generated by $\tilde{h}_{t}$ has the desired properties.

Proposition 3.2. Let $\psi \in \operatorname{Ham}(M, \omega), \tilde{\psi} \in \operatorname{Ham}(\tilde{M}, \tilde{\omega})$ be a lift given by the previous lemma and $x \in \operatorname{int}(P) \subset \operatorname{int}(\tilde{P})$. Then

(i) $\sharp\left(\psi\left(T_{x}\right) \cap R\right)=r \Rightarrow \sharp\left(\tilde{\psi}\left(\tilde{T}_{x}\right) \cap \tilde{R}\right)=r 2^{N-n}$.

(ii) $\psi\left(T_{x}\right) \cap T_{x}=\emptyset \Rightarrow \tilde{\psi}\left(\tilde{T}_{x}\right) \cap \tilde{T}_{x}=\emptyset$.

Moreover,

(iii) $\sharp\left(\psi\left(T_{x}\right) \pitchfork T_{x}\right)=r \Rightarrow \exists \tilde{\varphi} \in \operatorname{Ham}(\tilde{M}, \tilde{\omega})$ such that $\sharp\left(\tilde{\varphi}\left(\tilde{T}_{x}\right) \pitchfork \tilde{T}_{x}\right)=r 2^{N-n}$.

Remark 3.3. McDuff's method of probes [17] can be seen as a particular case of (ii).

Proof. To prove (i), suppose that $\tilde{q} \in \tilde{\psi}\left(\tilde{T}_{x}\right) \cap \tilde{R}$ and let $\tilde{p}=\tilde{\psi}^{-1}(\tilde{q}) \in \tilde{T}_{x}$. Then $p=\pi(\tilde{p}) \in T_{x}$ and

$$
\psi(p)=\psi(\pi(\tilde{p}))=\pi(\tilde{\psi}(\tilde{p}))=\pi(\tilde{q})=\pi(\tilde{\tau}(\tilde{q}))=\tau(\pi(\tilde{q}))=\tau(q)=\tau(\psi(p)) .
$$

Hence $q=\psi(p) \in R$, and so $q \in \psi\left(T_{x}\right) \cap R$.

Moreover, given $q \in \psi\left(T_{x}\right) \cap R$, let $\tilde{q} \in \pi^{-1}(q) \cap \tilde{R}$. Let $p=\psi^{-1}(q) \in T_{x}$ and $\tilde{p}=\tilde{\psi}^{-1}(\tilde{q})$. Then

$$
\begin{aligned}
& \pi(\tilde{p})=\pi\left(\tilde{\psi}^{-1}(\tilde{q})\right)=\psi^{-1}(\pi(\tilde{q}))=\psi^{-1}(q)=p \\
\Rightarrow & \tilde{p} \in \pi^{-1}(p) \subset \pi^{-1}\left(T_{x}\right)=\tilde{T}_{x} \\
\Rightarrow & \tilde{q}=\tilde{\psi}(\tilde{p}) \in \tilde{\psi}\left(\tilde{T}_{x}\right) .
\end{aligned}
$$

Hence $\tilde{q} \in \tilde{\psi}\left(\tilde{T}_{x}\right) \cap \tilde{R}$, and there are exactly $2^{N-n}$ such $\tilde{q}$ 's.

To prove (ii), suppose that $\tilde{q} \in \tilde{\psi}\left(\tilde{T}_{x}\right) \cap \tilde{T}_{x}$ and let $\tilde{p}=\tilde{\psi}^{-1}(\tilde{q}) \in \tilde{T}_{x}$. Then $p=\pi(\tilde{p}), q=\pi(\tilde{q}) \in T_{x}$ and

$$
\psi(p)=\psi(\pi(\tilde{p}))=\pi(\tilde{\psi}(\tilde{p}))=\pi(\tilde{q})=q,
$$

which implies that $q \in \psi\left(T_{x}\right) \cap T_{x}$.

To prove (iii), note that since $T_{x}$ and $\psi\left(T_{x}\right)$ intersect transversely at $r$ points in $M$, say $p_{1}, \ldots, p_{r} \in M$, we have that $\tilde{T}_{x}$ and $\tilde{\psi}\left(\tilde{T}_{x}\right)$ intersect in $Z$ in a Morse-Bott way along the $r$ orbits of the subtorus $K \subset \tilde{\mathbb{T}}$ given by $\pi^{-1}\left(p_{1}\right), \ldots, \pi^{-1}\left(p_{r}\right) \subset$ $\tilde{M}$. Standard equivariant neighborhood theorems in symplectic geometry imply that a sufficiently small neighborhood $\tilde{\mathcal{U}} \subset \tilde{M}$ of each of these isotropic tori is $K$-equivariantly symplectomorphic to $\mathcal{V}_{1} \times \mathcal{V}_{2} \subset\left(\mathbb{R}^{2 n}, \omega_{\text {st }}\right) \times\left(T^{*} K, \omega_{\text {can }}\right)$, where $\mathcal{V}_{1} \subset \mathbb{R}^{2 n}$ is a neighborhood of the origin, $\mathcal{V}_{2} \subset T^{*} K$ is a neighborhood of the 0 -section, and

$$
\omega_{\mathrm{st}}=d u \wedge d v=\sum_{j=1}^{n} d u_{j} \wedge d v_{j} \quad \text { and } \quad \omega_{\text {can }}=-d \lambda_{\text {can }}
$$

are the usual symplectic forms on $\mathbb{R}^{2 n}$ and $T^{*} K$, respectively. Moreover, we can identify $\mathcal{V}_{1}$ with a neighborhood $\mathcal{U} \subset M$ of the point in $\psi\left(T_{x}\right) \pitchfork T_{x}$ under consideration and require that

$$
T_{x} \cap \mathcal{U} \cong\left\{(u, v) \in \mathcal{V}_{1}: v=0\right\} \quad \text { and } \quad \psi\left(T_{x}\right) \cap \mathcal{U} \cong\left\{(u, v) \in \mathcal{V}_{1}: u=0\right\}
$$


Let $\varphi \in \operatorname{Ham}^{c}\left(\mathcal{V}_{2}, \omega_{\text {can }}\right)$ be such that $\sharp(\varphi(0$-section $) \pitchfork(0$-section $))=2^{N-n}$ (one can clearly construct such optimal displacing Hamiltonians supported in arbitrarily small neighborhoods of the 0 -section in $\left.T^{*} K\right)$. We can then consider id $\times \varphi$ : $\mathcal{V}_{1} \times \mathcal{V}_{2} \rightarrow \mathcal{V}_{1} \times \mathcal{V}_{2}$, extend as the identity to $\tilde{M}$ and compose with $\tilde{\psi}$ to obtain a Hamiltonian that perturbs the relevant intersection $K$-orbit into $2^{N-n}$ transversal intersection points. By doing that at each of the $r$ points in $\psi\left(T_{x}\right) \pitchfork T_{x}$ we obtain the desired $\tilde{\varphi} \in \operatorname{Ham}(\tilde{M}, \tilde{\omega})$.

\section{Corollary 3.4.}

(i) If $\sharp\left(\tilde{\psi}\left(\tilde{T}_{x}\right) \cap \tilde{R}\right) \geq m$ for any $\tilde{\psi} \in \operatorname{Ham}(\tilde{M}, \tilde{\omega})$, then

$$
\sharp\left(\psi\left(T_{x}\right) \cap R\right) \geq \frac{m}{2^{N-n}}, \forall \psi \in \operatorname{Ham}(M, \omega) .
$$

(ii) If $\tilde{T}_{x} \subset(\tilde{M}, \tilde{\omega})$ is non-displaceable, then $T_{x} \subset(M, \omega)$ is also non-displaceable.

(iii) If $\sharp\left(\tilde{\psi}\left(\tilde{T}_{x}\right) \pitchfork \tilde{T}_{x}\right) \geq m$ for any $\tilde{\psi} \in \operatorname{Ham}(\tilde{M}, \tilde{\omega})$, then

$$
\sharp\left(\psi\left(T_{x}\right) \pitchfork T_{x}\right) \geq \frac{m}{2^{N-n}}, \forall \psi \in \operatorname{Ham}(M, \omega) .
$$

Remark 3.5. This idea of using symplectic reduction to prove intersection properties of Lagrangian submanifolds was used by Tamarkin in [20]. It is also present in the work of Borman [6] on reduction properties of quasi-morphisms and quasi-states (see also [7]).

3.6. Symplectic reduction construction of toric manifolds. Recall that any symplectic toric manifold $\left(M^{2 n}, \omega\right)$ can be constructed as a symplectic reduction of

$$
\left(\tilde{M}=\mathbb{R}^{2 d} \cong \mathbb{C}^{2 d}, \tilde{\omega}=\sum_{j=1}^{d} d x_{j} \wedge d y_{j}\right),
$$

where $d$ is the number of facets of the corresponding polytope $P \subset\left(\mathbb{R}^{n}\right)^{*}$. This reduction is with respect to the natural action of a subtorus $K \subset \tilde{\mathbb{T}}=\mathbb{T}^{d}$ of dimension $d-n$, whose Lie algebra $\operatorname{Lie}(K) \subset \mathbb{R}^{d}=\operatorname{Lie}\left(\mathbb{T}^{d}\right)$ is determined as the kernel of the linear map

$$
\beta: \mathbb{R}^{d} \rightarrow \mathbb{R}^{n}, \quad \beta\left(e_{j}\right)=\nu_{j}, j=1, \ldots, d,
$$

where $\left\{e_{1}, \ldots, e_{d}\right\}$ is the canonical basis of $\mathbb{R}^{d}$ and $\nu_{1}, \ldots, \nu_{d} \in \mathbb{Z}^{n} \subset \mathbb{R}^{n}$ are the primitive integral interior normals to the facets of the moment polytope $P$.

When $K=K_{1} \times K_{2} \subset \mathbb{T}^{d}$, corresponding to a splitting $\operatorname{Lie}(K)=\operatorname{Lie}\left(K_{1}\right) \times$ Lie $\left(K_{2}\right) \subset \mathbb{R}^{d}$, recall that the principle of reduction in stages tells us that, at appropriate level sets, reduction with respect to the action of $K \subset \mathbb{T}^{d}$ is equivalent to

- first reducing with respect to $K_{1} \subset \mathbb{T}^{d}$, obtaining a symplectic manifold $\left(M_{1}, \omega_{1}\right)$ with Hamiltonian action of $\mathbb{T}^{d} / K_{1}$,

- then reducing $\left(M_{1}, \omega_{1}\right)$ with respect to $K_{2} \subset \mathbb{T}^{d} / K_{1}$.

This principle will be used repeatedly in the applications considered in the next sections. It is also the main ingredient in the proof of the following proposition, which in turn will be used in the proof of Theorem4.8 (cf. Proposition 4.9] in $\S$ 4.7).

Proposition 3.7. Let $\left(M^{2 n}, \omega\right)$ be a symplectic toric manifold and $\nu_{1}, \ldots, \nu_{d} \in \mathbb{Z}^{n}$ the primitive integral interior normals to the facets of its moment polytope $P \subset$ 
$\left(\mathbb{R}^{n}\right)^{*}$. Let $m_{1}, \ldots, m_{d} \in \mathbb{N}$ be such that

$$
\sum_{j=1}^{d} m_{j} \nu_{j}=0 .
$$

Then $\left(M^{2 n}, \omega\right)$ can be obtained as a symplectic reduction of the weighted projective space $\mathbb{C P}\left(m_{1}, \ldots, m_{d}\right)$.

Proof. $\left(M^{2 n}, \omega\right)$ can be obtained as the symplectic reduction of $\left(\mathbb{R}^{2 d}, d x \wedge d y\right)$ by the action of a subtorus $K \subset \mathbb{T}^{d}$ with $\operatorname{Lie}(K)=\operatorname{ker} \beta$, where the linear map $\beta$ is given by (5). This means in particular that

$$
\left(k_{1}, \ldots, k_{d}\right) \in \operatorname{Lie}(K) \subset \mathbb{R}^{d} \Leftrightarrow \sum_{j=1}^{d} k_{j} \nu_{j}=0,
$$

which together with (6) implies that $K$ can be written as $K=K_{1} \times K_{2}$ with $\operatorname{Lie}\left(K_{1}\right)=\operatorname{span}\left\{\left(m_{1}, \ldots, m_{d}\right)\right\}$. Since the weighted projective space $\mathbb{C P}\left(m_{1}, \ldots, m_{d}\right)$ is obtained as the symplectic reduction of $\left(\mathbb{R}^{2 d}, d x \wedge d y\right)$ by the action of $K_{1}$, one can use the principle of reduction in stages to conclude the proof.

\section{First application: Monotone Cases}

We will use the following results stated in Theorem 1.3

(i) The Clifford torus $T_{0}^{n} \subset \mathbb{C P}^{n}$ is non-displaceable and $\sharp\left(\psi\left(T_{0}^{n}\right) \pitchfork T_{0}^{n}\right) \geq$ $2^{n}, \forall \psi \in \operatorname{Ham}\left(\mathbb{C P}^{n}, \omega_{F S}\right)$ [5] 8].

(ii) The pair $\left(\mathbb{R P}^{2 n-1}, T_{0}^{2 n-1}\right)$ is non-displaceable in $\mathbb{C P} P^{2 n-1}$ and $\sharp\left(\psi\left(T_{0}^{2 n-1}\right) \pitchfork\right.$ $\left.\mathbb{R P}^{2 n-1}\right) \geq 2^{n}$ for any $\psi \in \operatorname{Ham}\left(\mathbb{C P}^{2 n-1}\right)[1]$.

Definition 4.1. Recall from Example 1.2 that any monotone toric symplectic manifold has a unique monotone torus fiber, called the centered or special torus fiber, which is the Lagrangian torus orbit over the "center" of its moment polytope. A symplectic reduction of a monotone toric symplectic manifold at a level containing its centered torus fiber, i.e. through the special "center" of its moment polytope, will be called a centered symplectic reduction.

4.2. Application 1. One can easily extend Alston's result to $\mathbb{C P}^{2 n}$. In fact, $\mathbb{C P}^{2 n}$ can be obtained as a centered symplectic reduction of $\mathbb{C P}^{2 n+1}$ (cf. Figure 1 for the $n=1$ case), and we get that

$$
\sharp\left(\psi\left(T_{0}^{2 n}\right) \pitchfork \mathbb{R} \mathrm{P}^{2 n}\right) \geq \frac{2^{n+1}}{2}=2^{n} \quad \text { for any } \psi \in \operatorname{Ham}\left(\mathbb{C P}^{2 n}\right) .
$$

Note that the fact that this estimate is known to be optimal for $\mathbb{C P}^{2}$ implies that Alston's bound for $\mathbb{C P}^{3}$ is also optimal.

4.3. Application 2. Let $\left(M^{2 n}, \omega\right)$ be a monotone toric manifold, $R$ its real part and $T$ its special centered torus fiber. Let $\nu_{1}, \ldots, \nu_{d} \in \mathbb{Z}^{n}$ denote the primitive integral interior normals to the facets of the moment polytope of $M$.

Proposition 4.4. If $\sum_{i=1}^{d} \nu_{i}=0$, then $T$ and the pair $(R, T)$ are non-displaceable. Moreover,

$$
\sharp(\psi(T) \pitchfork T) \geq 2^{n}, \forall \psi \in \operatorname{Ham}(M, \omega) .
$$




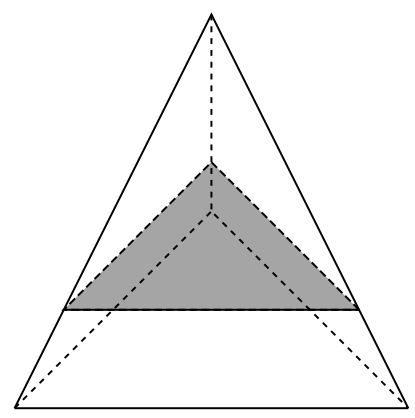

Figure 1. $\mathbb{C P}^{2}$ as a reduction of $\mathbb{C P}^{3}$.

Proof. Proposition 3.7 and the zero-sum condition on the normals imply that the standard symplectic reduction construction of $M$ from $\mathbb{C}^{d}$ factors through $\mathbb{C P}^{d-1}$. The monotone condition implies that this is a centered symplectic reduction and we can apply Corollary 3.4 .

4.5. Application 3. The monotone $M=\mathbb{C P}^{2} \sharp 3 \overline{\mathbb{C P}}^{2}$, i.e. the monotone blow-up of $\mathbb{C P}^{2}$ at three points, fits the context of Application 2 and can be obtained as a centered symplectic reduction of $\mathbb{C P}^{5}$. This means that

$$
\sharp(\psi(T) \pitchfork R) \geq \frac{2^{3}}{2^{3}}=1 \quad \text { for any } \psi \in \operatorname{Ham}(M) .
$$

It can also be obtained as a centered symplectic reduction of $\mathbb{C P}^{2} \times \mathbb{C P}^{2}$. Since our Floer combinatorial invariant of $\mathbb{C P}^{2} \times \mathbb{C P}^{2}$ is 4 , this gives the same bound:

$$
\sharp(\psi(T) \pitchfork R) \geq \frac{4}{2^{2}}=1 \quad \text { for any } \psi \in \operatorname{Ham}(M) .
$$

However, if one sees $M$ as a centered symplectic reduction of $\mathbb{C P}^{1} \times \mathbb{C P}^{1} \times \mathbb{C P}^{1}$, cf. Figure 2, one improves the bound to

$$
\sharp(\psi(T) \pitchfork R) \geq \frac{2^{3}}{2}=4 \quad \text { for any } \psi \in \operatorname{Ham}(M),
$$

which is optimal and coincides with the value of the Floer combinatorial invariant of the hexagon.
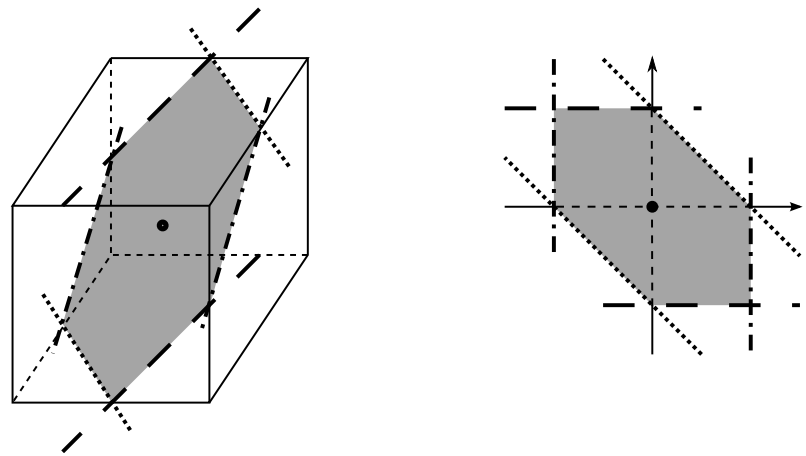

Figure 2. $\mathbb{C P} P^{2} \sharp 3 \overline{\mathbb{C P}}^{2}$ as a reduction of $\mathbb{C P}^{1} \times \mathbb{C P}^{1} \times \mathbb{C P}^{1}$. 
Let us describe the details of this reduction construction. The cube on the left of Figure 2, corresponding to the moment polytope of a monotone $\mathbb{C P}^{1} \times \mathbb{C P}^{1} \times \mathbb{C P}^{1}$, can be described by the following inequalites:

$$
\begin{array}{rrrr}
x_{1}+1 \geq 0, & x_{2}+1 \geq 0, & x_{3}+1 \geq 0, \\
-x_{1}+1 \geq 0, & -x_{2}+1 \geq 0, & -x_{3}+1 \geq 0 .
\end{array}
$$

The monotone $\mathbb{C P}^{2} \sharp 3 \overline{\mathbb{C P}}^{2}$ can be obtained from this toric manifold by centered symplectic reduction with respect to the circle $S^{1} \subset \mathbb{T}^{3}$ determined by the Lie algebra vector $(-1,-1,1) \in \mathbb{R}^{3}=\operatorname{Lie}\left(\mathbb{T}^{3}\right)$ at level $-x_{1}-x_{2}+x_{3}=0$. The quotient 2 -torus $\mathbb{T}^{3} / S^{1}$ acts on the reduced manifold and, with respect to its Lie algebra basis given by $(1,0,0),(0,1,0) \in \operatorname{Lie}\left(\mathbb{T}^{3} / S^{1}\right) \cong \mathbb{R}^{3} /\{(-1,-1,1)\}$, the resulting moment polytope is described by the above inequalities with $x_{3}=x_{1}+x_{2}$, i.e. the hexagon on the right of Figure 2 ,

In fact, the monotone $\mathbb{C P}^{2} \sharp 3 \overline{\mathbb{C P}}^{2}$ is just a particular case of the following more general proposition.

Proposition 4.6. Let $\left(M^{2 n}, \omega\right)$ be a monotone toric manifold, $R$ its real part and $T$ its special centered torus fiber. Suppose that the corresponding moment polytope $P \subset \mathbb{R}^{n}$ is symmetric; i.e. if $\nu \in \mathbb{Z}^{n}$ is the interior normal to a facet of $P$, then $-\nu$ is also the interior normal to a facet of $P$. Then

$$
\sharp(\psi(T) \pitchfork R) \geq 2^{n} \quad \text { for any } \psi \in \operatorname{Ham}(M) \text {, and this bound is optimal. }
$$

Proof. The fact that the polytope $P$ is symmetric implies that $M$ can be obtained as a symplectic reduction of the product of $d$ copies of $\mathbb{C P}^{1}$, where $2 d$ is the number of facets of $P$. The fact that $M$ is monotone implies that all the $\mathbb{C P}^{1}$ 's have the same area and that this is a centered symplectic reduction. Hence, we get from Corolary 3.4 that

$$
\sharp(\psi(T) \pitchfork R) \geq \frac{2^{d}}{2^{d-n}}=2^{n}, \forall \psi \in \operatorname{Ham}(M, \omega) .
$$

Since $\sharp(T \pitchfork R)=2^{n}$ the bound is indeed optimal.

4.7. Application 4. Let $\left(M^{2 n}, \omega\right)$ be a compact monotone symplectic toric manifold and $T$ its special centered torus fiber. Denote by $\nu_{1}, \ldots, \nu_{d} \in \mathbb{Z}^{n}$ the primitive integral interior normals to the facets of its Delzant polytope $P \subset\left(\mathbb{R}^{n}\right)^{*}$.

Lemma 4.8. There exists $k \in\{1, \ldots, d\}$ such that

$$
\nu_{k}+\sum_{j=1, j \neq k}^{d} m_{j} \nu_{j}=0
$$

with all $m_{j} \in \mathbb{N}$.

Proof. Since $P$ is the moment polytope of a compact toric manifold, the support of its associated fan is the whole $\mathbb{R}^{n}$. In particular, every lattice vector $\nu \in \mathbb{Z}^{n}$ belongs to a cone of the fan determined by some vertex of $P$, which means that it can be written as a non-negative integral linear combination of the primitive 
integral interior normals to the $n$ facets that meet at that vertex. The lemma follows by taking

$$
\nu=-\sum_{j=1}^{d} \nu_{j}
$$

Proposition 4.9. On any compact monotone toric symplectic manifold $(M, \omega)$ the special centered Lagrangian torus fiber $T$ is non-displaceable. Moreover,

$$
\sharp(\psi(T) \pitchfork T) \geq 2^{n}, \forall \psi \in \operatorname{Ham}(M, \omega) .
$$

Proof. Using the previous lemma, and after a possible re-ordering of the normals, we can assume that

$$
\sum_{j=1}^{d-1} m_{j} \nu_{j}+\nu_{d}=0 \quad \text { with } m_{1}, \ldots, m_{d-1} \in \mathbb{N} .
$$

This condition and Proposition 3.7 imply that the standard symplectic reduction construction of $M$ from $\mathbb{C}^{d}$ factors through the weighted projective space $\mathbb{C P}\left(1, m_{1}, \ldots, m_{d-1}\right)$. The monotone condition implies that this factorization goes through the special centered non-displaceable torus fiber of this weighted projective space (cf. Theorem 1.7) and we can apply Corollary 3.4.

As a particular example, consider the monotone $M=\mathbb{C P}^{2} \sharp \overline{\mathbb{C P}}^{2}$, i.e. the monotone blow-up of $\mathbb{C P}^{2}$ at one point, with polytope $P \subset\left(\mathbb{R}^{2}\right)^{*}$ given by

$$
\begin{array}{rrrr}
x_{1}+1 \geq 0 & \left(\nu_{1}=(1,0)\right), & -x_{1}-x_{2}+1 \geq 0 & \left(\nu_{3}=(-1,-1)\right), \\
x_{2}+1 \geq 0 & \left(\nu_{2}=(0,1)\right), & x_{1}+x_{2}+1 \geq 0 & \left(\nu_{4}=(1,1)\right) .
\end{array}
$$

We have that

$$
\left(\nu_{1}+\nu_{2}+2 \nu_{3}\right)+\nu_{4}=0
$$

and $M$ can be obtained as a "centered" symplectic reduction of $\mathbb{C P}(1,1,1,2)$. In fact, the polytope of $\mathbb{C P}(1,1,1,2)$ is given by

$$
\begin{aligned}
& x_{1}+1 \geq 0, \\
& x_{3}+1 \geq 0, \\
& x_{2}+1 \geq 0 \text {, } \\
& -x_{1}-x_{2}-2 x_{3}+1 \geq 0 \text {, }
\end{aligned}
$$

and its reduction with respect to the circle $S^{1} \subset \mathbb{T}^{3}$ determined by $(1,1,1) \in \mathbb{R}^{3}=$ $\operatorname{Lie}\left(\mathbb{T}^{3}\right)$ at level

$$
x_{1}+x_{2}+x_{3}=0 \text {, i.e. } x_{3}=-x_{1}-x_{2},
$$

gives rise to the above polytope for $M=\mathbb{C P}^{2} \sharp \overline{\mathbb{C P}}^{2}$.

\section{Second application: Non-monotone Fano CASES}

For the applications in this section we will assume that some form of the following general result is true:

- If $T_{i}$ or the pair $\left(T_{i}, R_{i}\right)$ have $H F \neq 0$ or are non-displaceable in $\left(M_{i}, \omega_{i}\right)$, $i=1,2$, then the same is true for the corresponding $T_{1} \times T_{2}$ and $\left(T_{1} \times\right.$ $\left.T_{2}, R_{1} \times R_{2}\right)$ in $\left(M_{1} \times M_{2}, \omega_{1} \times \omega_{2}\right)$.

Remark 5.1. For Lagrangian torus orbits in toric symplectic manifolds, the set-up of Woodward 22] applies and proves a result of this form (cf. 21]). 
Moreover, in some of the applications below we will also use the following result:

- In the total space of the line bundle $\mathcal{O}(-1) \rightarrow \mathbb{C P}^{1}$, the special torus $T$ sitting over the origin in the polygon on the right side of Figure 3 is nondisplaceable. This has been proved by Woodward (cf. Example 1.3 in [22]) and can also be seen as a consequence of a result of Cho in 9] (cf. the polygon on the left side of Figure 3).
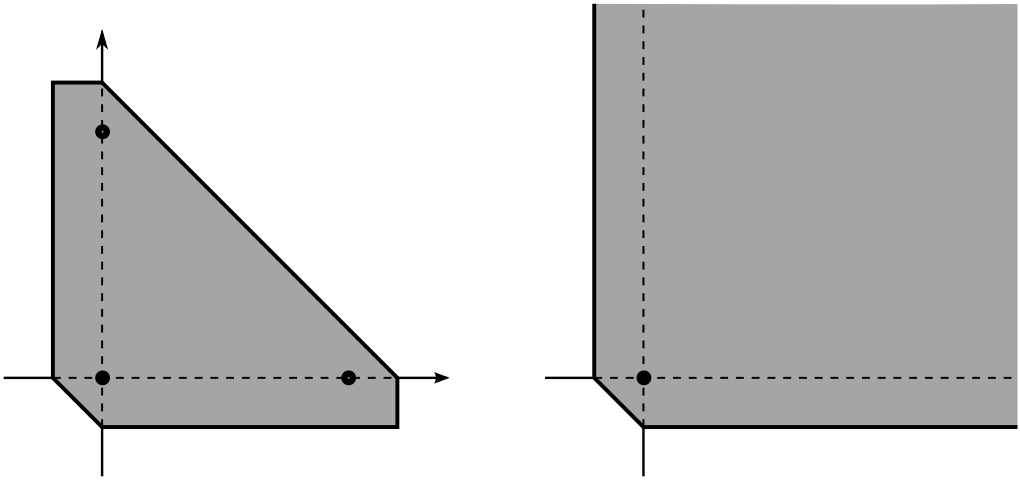

Figure 3. Cho's result on the left and its limit on the right, giving a non-displaceable torus fiber in the total space of the line bundle $\mathcal{O}(-1) \rightarrow \mathbb{C P}^{1}$.

5.2. Application 5. Consider $M=\mathbb{C P}^{2} \sharp \overline{\mathbb{C P}}^{2}$, i.e. the blow-up of $\mathbb{C P}^{2}$ at one point.

Figure 4 illustrates how one can obtain two non-displaceable torus fibers when the exceptional divisor is small, i.e. smaller than monotone. On the left, one thinks of $M$ as a symplectic reduction of $\mathbb{C P}^{2} \times \mathbb{C P}^{1}$, with $\mathbb{C P}^{2}$ "smaller" than $\mathbb{C P}^{1}$, to show that the torus fiber over the origin is non-displaceable. On the right, one thinks of $M$ as a symplectic reduction of $\mathcal{O}(-1) \times \mathbb{C P}^{2}$ to show that a torus fiber "close" to the exceptional divisor is non-displaceable.

Figure 5 illustrates how one can obtain one non-displaceable torus fiber when the exceptional divisor is big, i.e. bigger than monotone. One again thinks of $M$ as a symplectic reduction of $\mathbb{C P}^{2} \times \mathbb{C P}^{1}$, but now with $\mathbb{C P}^{2}$ "bigger" than $\mathbb{C P}^{1}$.

Note that the monotone case with just one non-displaceable torus fiber over the special central point can be obtained as a limit of any of these.

5.3. Application 6. A very similar idea applies to $M=\mathbb{C P}^{2} \sharp 2 \overline{\mathbb{C P}}^{2} \cong\left(\mathbb{C P}^{1} \times\right.$ $\left.\mathbb{C P}^{1}\right) \sharp \overline{\mathbb{C P}}^{2}$, i.e. the equal blow-up of $\mathbb{C P}^{2}$ at two points which can also be thought of as the blow-up of $\mathbb{C P}^{1} \times \mathbb{C P}^{1}$ at one point. One recovers the results of FukayaOh-Ohta-Ono [14, 15] and Woodward [22] illustrated in Figures 6] and 7.

Figure 6] illustrates how one can obtain two non-displaceable torus fibers in the big two-point blow-up of $\mathbb{C P}^{2}$, which is equivalent to a small one-point blowup of $\mathbb{C P}^{1} \times \mathbb{C P}^{1}$. On the left, one thinks of $M$ as a symplectic reduction of $\mathbb{C P}^{2} \times \mathbb{C P}^{1} \times \mathbb{C P}^{1}$, with "big" $\mathbb{C P}^{2}$, to show that the torus fiber over the origin is non-displaceable. On the right, one thinks of $M$ as a symplectic reduction of 

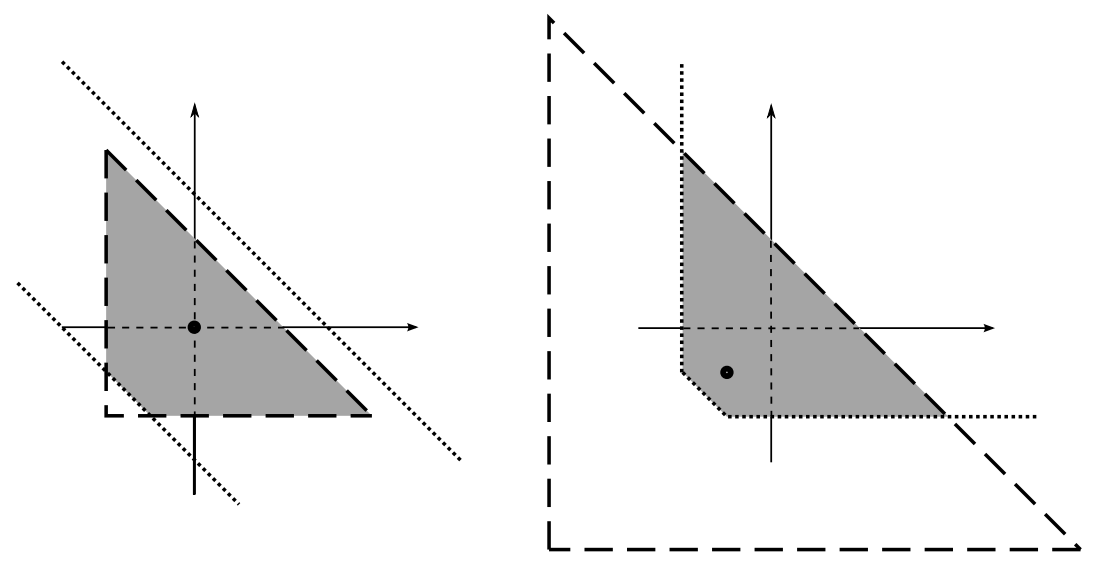

Figure 4. $\mathbb{C P}^{2} \sharp \overline{\mathbb{C P}}^{2}$ as a reduction of $\mathbb{C P}^{2} \times \mathbb{C P}^{1}$ (on the left, $\mathbb{C P}^{2}$ is "smaller" than $\mathbb{C P}^{1}$ ) and of $\mathcal{O}(-1) \times \mathbb{C P}^{2}$ (on the right).

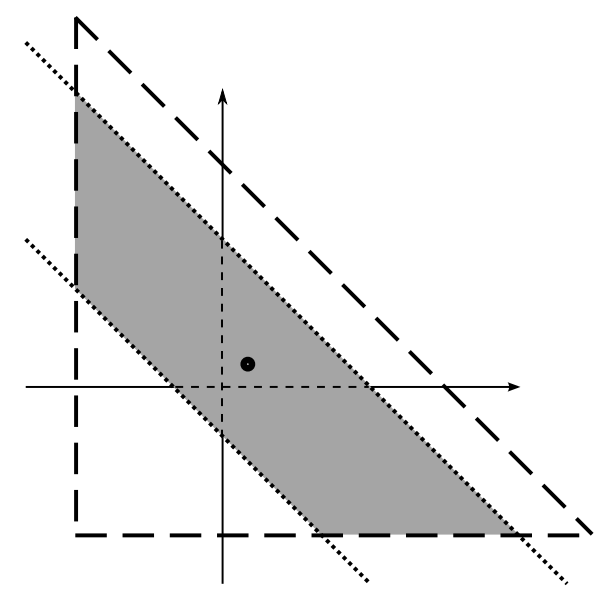

Figure 5. $\mathbb{C P}^{2} \sharp \overline{\mathbb{C P}}^{2}$ as a reduction of $\mathbb{C P}^{2} \times \mathbb{C P}^{1}$, now with $\mathbb{C P}^{2}$ "bigger" than $\mathbb{C P}^{1}$.

$\mathcal{O}(-1) \times \mathbb{C P}^{2}$ to show that a torus fiber "close" to the blown-up point on $\mathbb{C P}^{1} \times \mathbb{C P}^{1}$ is non-displaceable.

Figure 7 illustrates how one can obtain three non-displaceable torus fibers in the small two-point blow-up of $\mathbb{C P}^{2}$, which is equivalent to a big one-point blowup of $\mathbb{C P}^{1} \times \mathbb{C P}^{1}$. On the left, one thinks of $M$ as a symplectic reduction of $\mathbb{C P}^{2} \times \mathbb{C P}^{1} \times \mathbb{C P}^{1}$, with "small" $\mathbb{C P}^{2}$ and large $\mathbb{C P}^{1}$ 's, to show that a torus fiber "close" to the origin is non-displaceable. On the right, one thinks of $M$ as a symplectic reduction of $\mathcal{O}(-1) \times \mathbb{C P}^{1} \times \mathbb{C P}^{1}$ to show that there is a non-displaceable torus fiber "close" to each blown-up point on $\mathbb{C P}^{2}$.

Again, note that the monotone case with just one non-displaceable torus fiber over the special central point can be obtained as a limit of any of these. It could also be obtained using Proposition 4.9, as was illustrated in $\S 4.7$ for the monotone one-point blow-up $\mathbb{C P}^{2} \sharp \overline{\mathbb{C P}}^{2}$. 

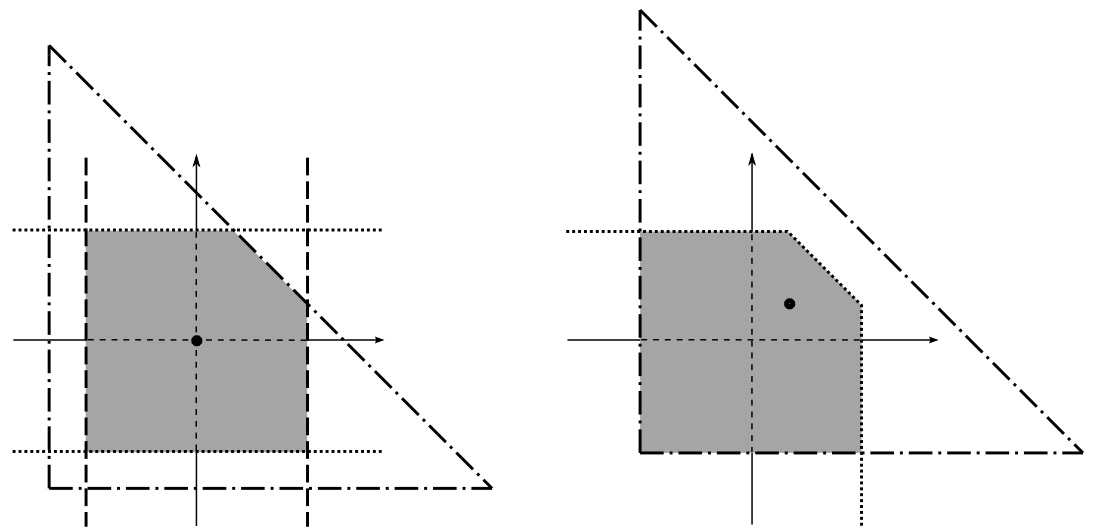

Figure 6. $\left(\mathbb{C P}^{1} \times \mathbb{C P}^{1}\right) \sharp \overline{\mathbb{C P}}^{2}$ (small blow-up), equivalently $\mathbb{C P}^{2} \sharp 2 \overline{\mathbb{C P}}^{2}$ (big blow-ups), as a reduction of $\mathbb{C P}^{2} \times \mathbb{C P}^{1} \times \mathbb{C P}^{1}$ (on the left) and of $\mathcal{O}(-1) \times \mathbb{C P}^{2}$ (on the right).
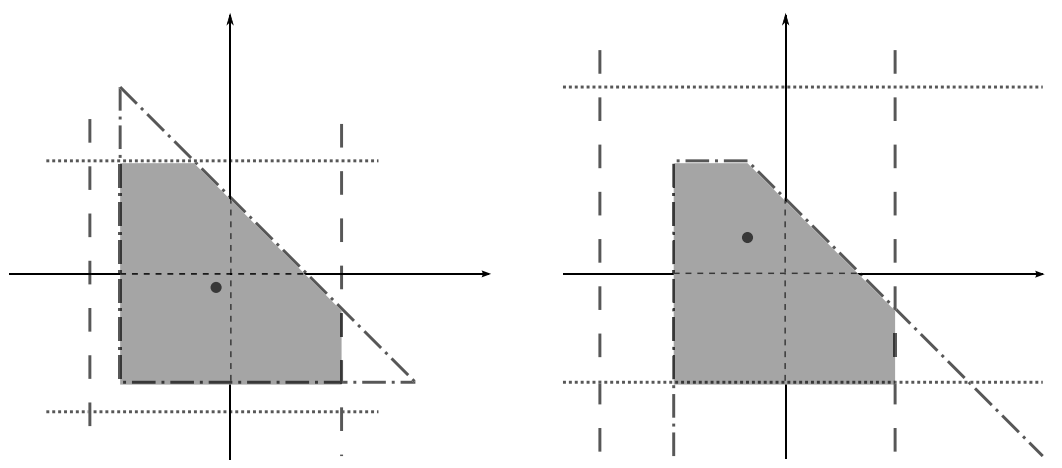

Figure 7. $\left(\mathbb{C P}^{1} \times \mathbb{C P}^{1}\right) \sharp \overline{\mathbb{C P}}^{2}$ (big blow-up), equivalently $\mathbb{C P}^{2} \sharp 2 \overline{\mathbb{C P}}^{2}$ (small blow-ups), as a reduction of $\mathbb{C P}^{2} \times \mathbb{C P}^{1} \times \mathbb{C P}^{1}$ (on the left) and of $\mathcal{O}(-1) \times \mathbb{C P}^{1} \times \mathbb{C P}^{1}$ (on the right).

5.4. Application 7. Here we will use the same idea to understand an example that Fukaya, Oh, Ohta and Ono [14, 15] presented in Figure 8 (see Example 10.3 in [16]). The symplectic manifold is $M=\mathbb{C P}^{2} \sharp 2 \overline{\mathbb{C P}}^{2}$ with blow-ups of different sizes, one smaller than monotone and the other bigger than monotone, and they obtain a closed interval of non-displaceable torus fibers. This can also be obtained by considering $M$ as the symplectic reduction of $\mathcal{O}(-1) \times \mathbb{C P}^{1} \times \mathbb{C P}^{1}$ (or the compact $\left.\left(\mathbb{C P}^{2} \sharp \overline{\mathbb{C P}}^{2}\right) \times \mathbb{C P}^{1} \times \mathbb{C P}^{1}\right)$, as shown in Figure 8 . 


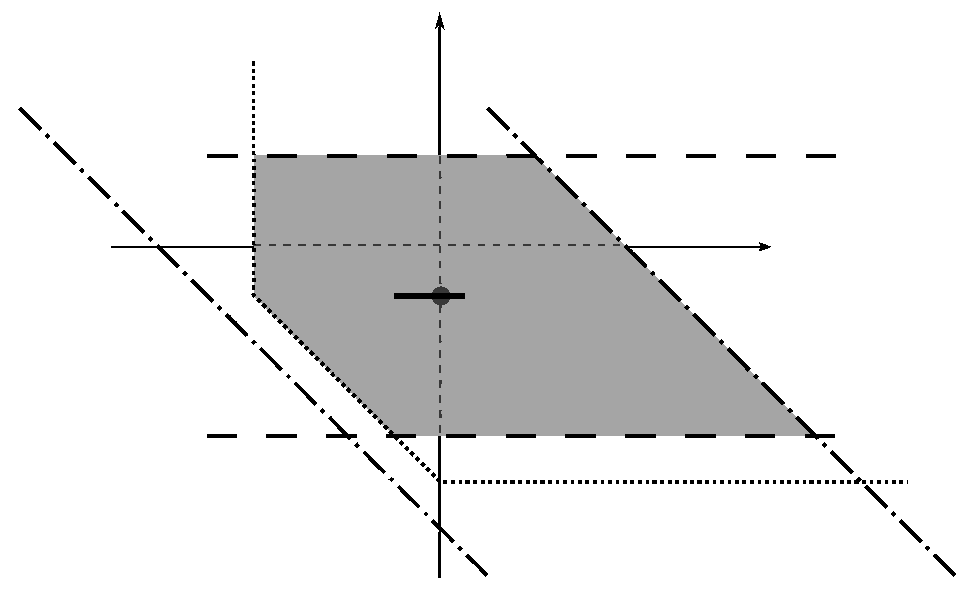

Figure 8. $\mathbb{C P}^{2} \sharp 2 \overline{\mathbb{C P}}^{2}$ as a reduction of $\mathcal{O}(-1) \times \mathbb{C P}^{1} \times \mathbb{C P}^{1}$.

The details are as follows. Consider $\left(\mathbb{C P}^{2} \sharp 2 \overline{\mathbb{C P}}^{2}, \omega_{\alpha}\right)$ given by the Delzant polytope $P_{\alpha} \subset\left(\mathbb{R}^{2}\right)^{*}$ determined by the following inequalities:

$$
\begin{array}{rlrl}
x_{1}+1 & \geq 0, & x_{1}+x_{2}+1+\alpha & \geq 0, \\
x_{2}+1 & \geq 0, & -x_{2}+(1-2 \alpha) & \geq 0, \\
-\left(x_{1}+x_{2}\right)+1 & \geq 0, & \text { with } 0<\alpha<1 .
\end{array}
$$

The non-displaceable torus fibers are over the points with coordinates $(-\alpha+\lambda,-\alpha)$ for $0<\lambda<3 \alpha / 2$ (Figure 8 corresponds to $\alpha=\lambda=1 / 4$ ). To prove this for each such pair of real numbers $\alpha$ and $\lambda$, we consider $\mathcal{O}(-1) \times \mathbb{C P}^{1} \times \mathbb{C P}^{1}$ with moment polytope given by the cartesian product of the following polytopes:

- the one for the $\mathcal{O}(-1)$ factor is given by the inequalities

$$
x_{1}+1 \geq 0, \quad x_{1}+x_{2}+1+\alpha \geq 0 \quad \text { and } \quad x_{2}+1+\lambda \geq 0,
$$

having a non-displaceable torus fiber over the point with coordinates $x_{1}=$ $-\alpha+\lambda$ and $x_{2}=-\alpha$;

- the one for the first $\mathbb{C P}^{1}$ factor is given by the inequalities

$$
x_{3}+1 \geq 0 \text { and }-x_{3}+(1-2 \alpha) \geq 0,
$$

having a non-displaceable torus fiber over the point with coordinate $x_{3}=$ $-\alpha$;

- the one for the second $\mathbb{C P}^{1}$ factor is given by the inequalities

$$
x_{4}+1+4 \alpha-2 \lambda \geq 0 \text { and }-x_{4}+1 \geq 0,
$$

having a non-displaceable torus fiber over the point with coordinate $x_{4}=$ $-2 \alpha+\lambda$.

We can now apply symplectic reduction with respect to the 2 -torus $\mathbb{T}^{2} \subset \mathbb{T}^{4}$ determined by the Lie algebra vectors $(0,-1,1,0),(-1,-1,0,1) \in \mathbb{R}^{4}=\operatorname{Lie}\left(\mathbb{T}^{4}\right)$ at the level given by

$$
x_{3}=x_{2} \quad \text { and } \quad x_{4}=x_{1}+x_{2}
$$

to obtain the polytope $P_{\alpha}$ with non-displaceable torus fiber over the point with coordinates $x_{1}=-\alpha+\lambda$ and $x_{2}=-\alpha$. 
5.5. Application 8. One can use the same idea to understand non-displaceable torus fibers on $M=\mathbb{C P}^{2} \sharp 3 \overline{\mathbb{C P}}^{2}$ for all possible sizes of blown-up points. Figure 9 illustrates the case of three small size blow-ups, where one gets four non-displaceable torus fibers.

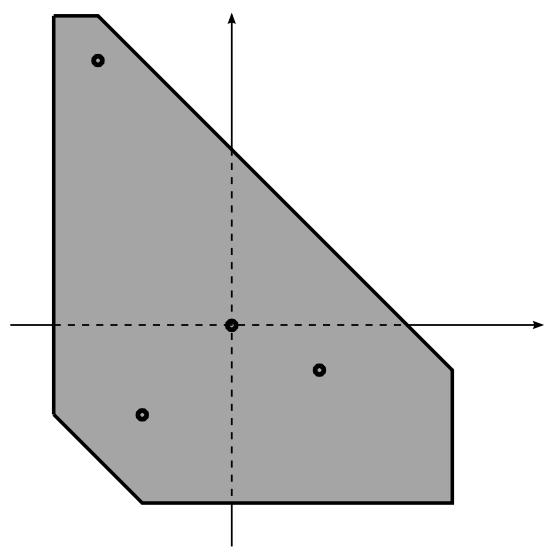

Figure 9. Four non-displaceable torus fibers on $\mathbb{C P}^{2} \sharp 3 \overline{\mathbb{C P}}^{2}$.

The center fiber is obtained by seeing $M$ as the symplectic reduction of $\mathbb{C P}^{2} \times$ $\mathbb{C P}^{1} \times \mathbb{C P}^{1} \times \mathbb{C P}^{1}$, as on the left side of Figure 10, while each of the off-center fibers is obtained by seeing $M$ as the symplectic reduction of $\mathcal{O}(-1) \times \mathbb{C P}^{1} \times \mathbb{C P}^{1} \times \mathbb{C P}^{1}$, as on the right side of Figure 10.
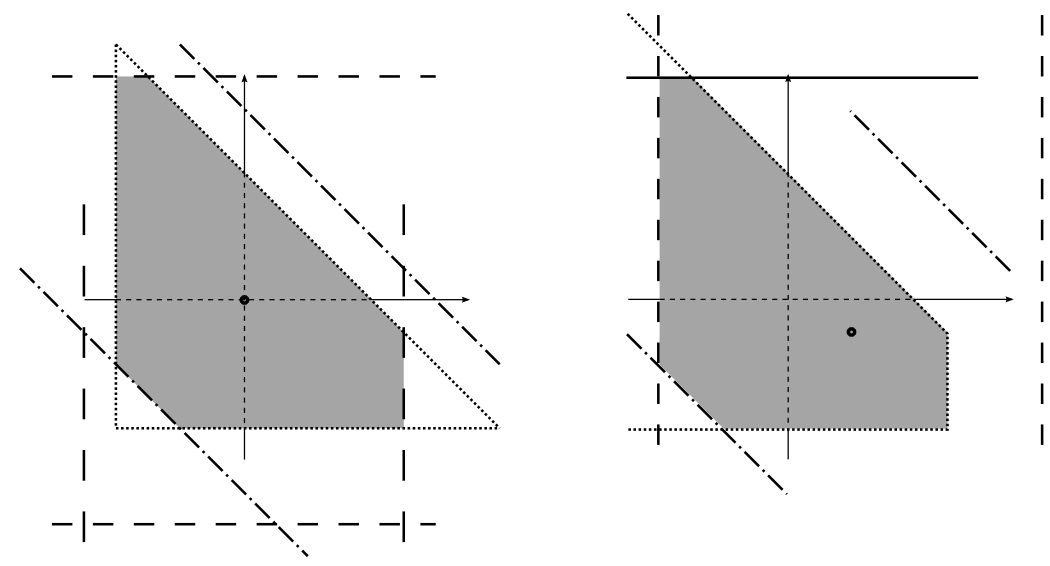

Figure 10. $\quad \mathbb{C P}^{2} \sharp 3 \overline{\mathbb{C P}}^{2}$ as a reduction of $\mathbb{C P}^{2} \times \mathbb{C P}^{1} \times \mathbb{C P}^{1} \times \mathbb{C P}^{1}$ (on the left) and of $\mathcal{O}(-1) \times \mathbb{C P}^{1} \times \mathbb{C P}^{1} \times \mathbb{C P}^{1}$ (on the right).

Note that we can also get a closed interval of non-displaceable torus fibers for $M=\mathbb{C P}^{2} \sharp 3 \overline{\mathbb{C P}}^{2}$, e.g. by blowing up the example in Application 6 at the lower right corner of Figure 8 


\section{Third application: Non-FAno CASES}

Here we will use the basic non-displaceability result for the central torus fiber of a weighted projective space stated in Theorem 1.7 and show how it implies nondisplaceability of at least one torus fiber on any Hirzebruch surface, a result of Fukaya-Oh-Ohta-Ono [14] (see Example 10.1 in [16]), and a continuous interval of non-displaceable torus fibers on a particular non-Fano toric surface considered by McDuff [18].

6.1. Application 9. Consider the weighted projective space $\mathbb{C P}(1,1, k)$, the symplectic quotient of $\mathbb{C}^{3}$ by the $S^{1}$-action with weights $(1,1, k)$, with moment polytope $P_{k} \subset \mathbb{R}^{2}$ given by

$$
x_{1}+1 \geq 0, \quad x_{2}+1 \geq 0 \quad \text { and } \quad-x_{1}-k x_{2}+1 \geq 0 .
$$

The special torus sitting over the origin is non-displaceable. Figure 11 illustrates the $k=2$ case.

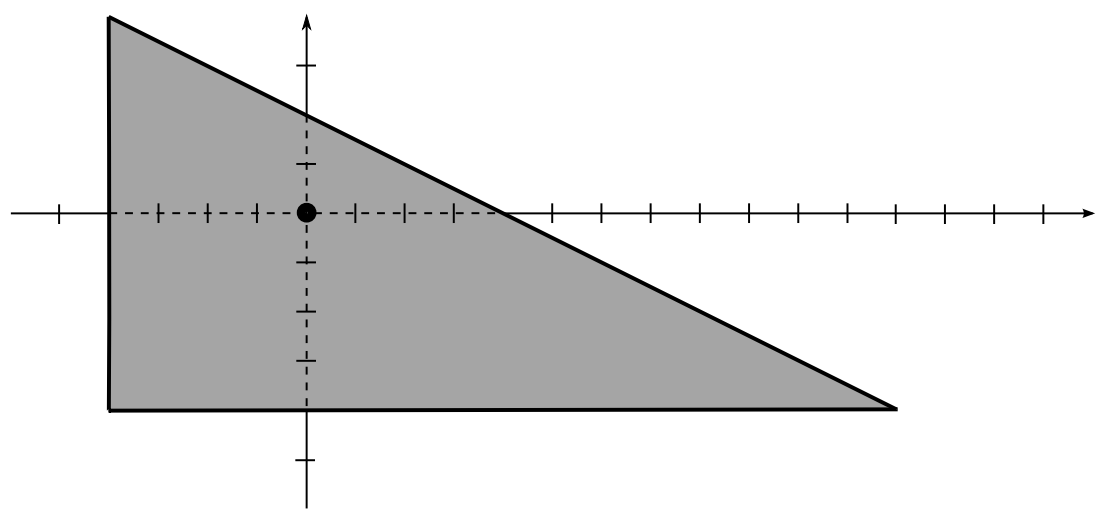

Figure 11. $\mathbb{C P}(1,1,2)$.

Now let $H_{k}:=\mathbb{P}(\mathcal{O}(-k) \oplus \mathbb{C}) \rightarrow \mathbb{C P}^{1}$ be a Hirzebruch surface, with $2 \leq k \in \mathbb{N}$. Each of these Hirzebruch surfaces can be seen as a centered symplectic reduction of $\mathbb{C P}(1,1, k) \times \mathbb{C P}^{1}$, and that implies at least one non-displaceable torus fiber on any $H_{k}$. Figure 12 illustrates the $k=2$ case.

6.2. Application 10. Here we will consider the non-Fano toric symplectic 4manifold described by McDuff in $\S 2.1$ of [18. We already pointed out its potential significance in Remark 1.10.

Consider the Delzant polytope $P \subset\left(\mathbb{R}^{2}\right)^{*}$ determined by the following inequalities:

$$
\begin{aligned}
x_{1}+1 & \geq 0, & -x_{1}-3 x_{2}+3 \geq 0, \\
x_{2}+1 & \geq 0, & -x_{1}-2 x_{2}+3 \geq 0 . \\
-x_{2}+1 & \geq 0, &
\end{aligned}
$$

The torus fibers over the points with coordinates $x_{1}=\lambda$ and $x_{2}=0$, with $1<\lambda<$ 2 , are non-displaceable by probes. We can show that all these fibers are in fact non-displaceable by considering this toric 4-manifold as a symplectic reduction of $\mathbb{C P}(1,1,2) \times \mathbb{C P}^{1} \times \mathcal{O}(-1)$, as shown in Figure 13 (where $\lambda=3 / 2$ ). 


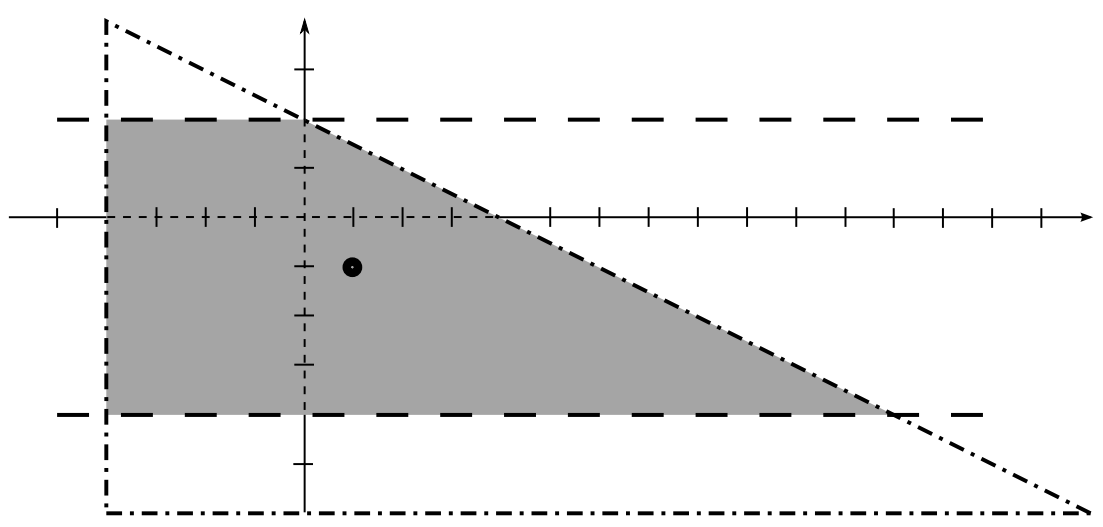

Figure 12. The Hirzebruch surface $H_{2}$ as a reduction of $\mathbb{C P}(1,1,2) \times \mathbb{C P}^{1}$.

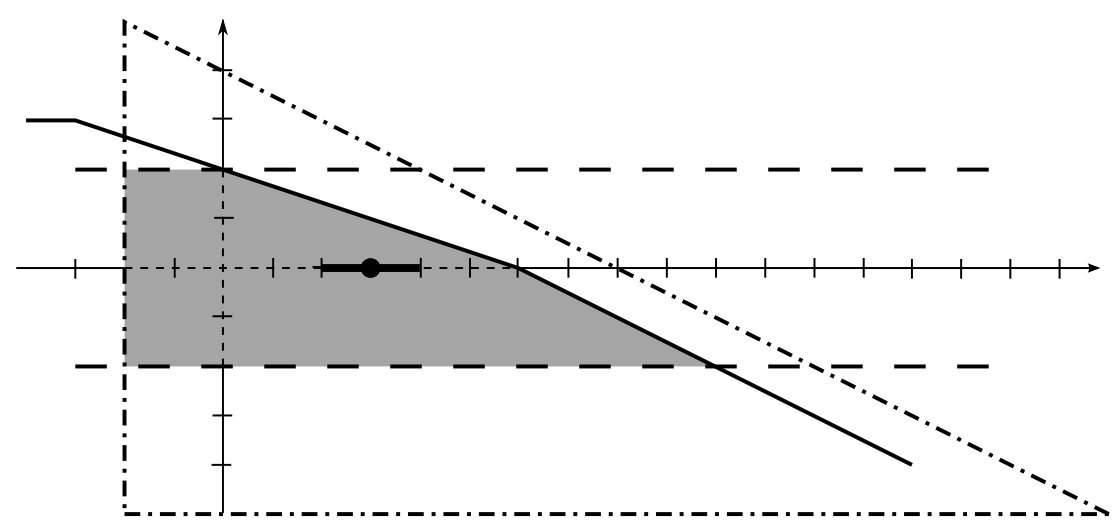

Figure 13. Non-Fano toric surface with an interval on nondisplaceable torus fibers.

More precisely, for each $1<\lambda<2$, consider $\mathbb{C P}(1,1,2) \times \mathbb{C P}^{1} \times \mathcal{O}(-1)$ with moment polytope given by the cartesian product of the following polytopes:

- the one for the $\mathbb{C P}(1,1,2)$ factor is given by the inequalities

$$
x_{1}+1 \geq 0, \quad x_{2}+1+\lambda \geq 0 \quad \text { and } \quad-x_{1}-2 x_{2}+1+2 \lambda \geq 0,
$$

having a non-displaceable torus fiber over the point with coordinates $x_{1}=\lambda$ and $x_{2}=0$;

- the one for the $\mathbb{C P}^{1}$ factor is given by the inequalities

$$
x_{3}+1 \geq 0 \text { and }-x_{3}+1 \geq 0,
$$

having a non-displaceable torus fiber over the point with coordinate $x_{3}=0$;

- the one for the $\mathcal{O}(-1)$ factor is given by the inequalities

$$
x_{4}+3 \geq 0, \quad x_{5}+3-\lambda \geq 0 \quad \text { and } \quad x_{4}+x_{5}+3 \geq 0,
$$

having a non-displaceable torus fiber over the point with coordinates $x_{4}=$ $-\lambda$ and $x_{5}=0$. 
We can now apply symplectic reduction with respect to the 3 -torus $\mathbb{T}^{3} \subset \mathbb{T}^{5}$ determined by the Lie algebra vectors $(0,-1,1,0,0),(-1,-2,0,1,0),(0,-1,0,0,1) \in$ $\mathbb{R}^{5}=\operatorname{Lie}\left(\mathbb{T}^{5}\right)$ at the level given by

$$
x_{3}=x_{2}, \quad x_{4}=-x_{1}-2 x_{2} \text { and } \quad x_{5}=-x_{2}
$$

to obtain the polytope $P$ with non-displaceable torus fiber over the point with coordinates $x_{1}=\lambda$ and $x_{2}=0$.

\section{ACKNOWLEDGEMENTS}

The authors thank Matthew Strom Borman, Cheol-Hyun Cho, Rui Loja Fernandes, Dusa McDuff and Chris Woodward for several useful discussions regarding this paper.

The authors also thank the IMPA and IST for their warm hospitality during the preparation of this work.

Part of these results were first presented by the first author at the Workshop on Symplectic Geometry and Topology, Kyoto University, Japan, February 14-18, 2011. The first author thanks the organizers, Kenji Fukaya and Kaoru Ono, for the opportunity to participate in such a wonderful event.

\section{RefEREnCES}

[1] G. Alston, Lagrangian Floer homology of the Clifford torus and real projective space in odd dimensions, Journal of Symplectic Geometry 9 (2011), 83-106. MR2787362

[2] G. Alston and L. Amorim, Floer cohomology of torus fibers and real Lagrangians in Fano toric manifolds, to appear in Internat. Math. Research Notices.

[3] P. Biran and O. Cornea, Lagrangian Quantum Homology, in "New perspectives and challenges in symplectic field theory", 1-44, CRM Proc. Lecture Notes 49, AMS, 2009. MR2555932 (2010m:53132)

[4] P. Biran and O. Cornea, Rigidity and uniruling for Lagrangian submanifolds, Geom. Topol. 13 (2009), 2881-2989. MR2546618 (2010k:53129)

[5] P. Biran, M. Entov and L. Polterovich, Calabi quasimorphisms for the symplectic ball, Comm. Contemp. Math. 6 (2004), 793-802. MR2100764 (2005f:53152)

[6] M. S. Borman, Symplectic reduction of quasi-morphisms and quasi-states, J. Symplectic Geom. 10 (2012), no. 2, 225-246. MR2926996

[7] M. S. Borman, Quasi-states, quasi-morphisms, and the moment map, preprint (2011), arXiv:1105.1805, to appear in Internat. Math. Research Notices.

[8] C.-H. Cho, Holomorphic discs, spin structures and the Floer cohomology of the Clifford torus, Int. Math. Res. Not. 35 (2004), 1803-1843. MR2057871 (2005a:53148)

[9] C.-H. Cho, Non-displaceable Lagrangian submanifolds and Floer cohomology with nonunitary line bundle, J. Geom. Phys. 58 (2008), 1465-1476. MR2463805 (2009j:53118)

[10] C.-H. Cho and M. Poddar, Holomorphic orbidiscs and Lagrangian Floer cohomology of symplectic toric orbifolds, preprint (2012), arXiv:1206.3994.

[11] C.-H. Cho and Y.-G. Oh, Floer cohomology and disc instantons of Lagrangian torus fibers in toric Fano manifolds, Asian J. Math. 10 (2006), 773-814. MR2282365 (2007k:53150)

[12] D. Cox, Lectures on Toric Varieties, http://www.cs.amherst.edu/ dac/lectures/coxcimpa. pdf.

[13] M. Entov and L. Polterovich, Rigid subsets of symplectic manifolds, Compositio Math. 145 (2009), 773-826. MR2507748(2011a:53174)

[14] K. Fukaya, Y.-G. Oh, H. Ohta and K. Ono, Lagrangian Floer theory on compact toric manifolds I, Duke Math. J. 151 (2010), 23-174. MR2573826(2011d:53220)

[15] K. Fukaya, Y.-G. Oh, H. Ohta and K. Ono, Lagrangian Floer theory on compact toric manifolds II: Bulk deformations, Selecta Math. 17 (2011), no. 3, 609-711. MR.2827178

[16] K. Fukaya, Y.-G. Oh, H. Ohta and K. Ono, Lagrangian Floer theory on compact toric manifolds: A survey, preprint (2010), arXiv:1011.4044. 
[17] D. McDuff, Displacing Lagrangian toric fibers via probes, Proc. Sympos. Pure Math., 82, Amer. Math. Soc., Providence, RI, 2011. MR2768658

[18] D. McDuff, The topology of toric symplectic manifolds, Geom. Topol. 15 (2011), 145-190. MR2776842

[19] Y. Oh, Floer cohomology of Lagrangian intersections and pseudo-holomorphic disks I, Comm. Pure Appl. Math. 46 (1993) 949-993; Addendum, Comm. Pure Appl. Math. 48 (1995), 12991302. MR 1223659 (95d:58029a)

[20] D. Tamarkin, Microlocal condition for non-displaceability, preprint (2008), arXiv:0809.1584.

[21] G. Wilson and C. Woodward, Quasimap Floer cohomology and singular symplectic quotients, preprint (2011), arXiv:1105.0712.

[22] C. Woodward, Gauged Floer theory of toric moment fibers, Geom. Funct. Anal. 21 (2011), no. 3, 680-749. MR2810861 (2012f:53187)

Centro de Análise Matemática, Geometria e Sistemas Dinâmicos, Departamento de Matemática, Instituto Superior Técnico, Av. Rovisco Pais, 1049-001 Lisboa, Portugal

E-mail address: mabreu@math.ist.utl.pt

Instituto de Matemática, Universidade Federal do Rio de Janeiro, Cidade UniverSITÁRIA, CEP 21941-909, RIO DE JANEIRO, BRAZIL

E-mail address: leonardo@impa.br 